\title{
Cygnus survey with the Giant Metrewave Radio Telescope at 325 and $610 \mathrm{MHz}$ : the catalog ${ }^{\star}$
}

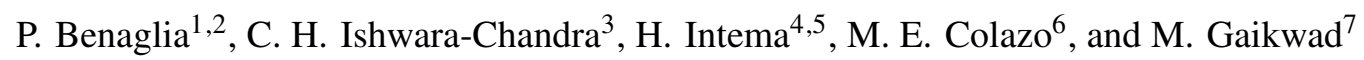 \\ 1 Instituto Argentino de Radioastronomía, CONICET \& CICPBA, CC5 (1897) Villa Elisa, Prov. de Buenos Aires, Argentina \\ e-mail: paula@iar.unlp.edu.ar \\ 2 Facultad de Ciencias Astronómicas y Geofísicas, UNLP, Paseo del Bosque s/n, 1900 La Plata, Argentina \\ 3 National Centre for Radio Astrophysics (NCRA-TIFR), Pune 411 007, India \\ 4 International Centre for Radio Astronomy Research, Curtin University, Bentley, WA 6102, Australia \\ ${ }^{5}$ Leiden Observatory, Leiden University, Niels Bohrweg 2, 2333 CA Leiden, The Netherlands \\ 6 Comisión Nacional de Actividades Espaciales, Paseo Colón 751, (1063) CABA, Argentina \\ 7 Max-Planck-Institut für Radioastronomie, Auf dem Hügel 69, 53121 Bonn, Germany
}

Received 8 March 2020 / Accepted 23 July 2020

\begin{abstract}
Context. Observations at the radio continuum band below the gigahertz band are key when the nature and properties of nonthermal sources are investigated because their radio radiation is strongest at these frequencies. The low radio frequency range is therefore the best to spot possible counterparts to very high-energy (VHE) sources: relativistic particles of the same population are likely to be involved in radio and high-energy radiation processes. Some of these counterparts to VHE sources can be stellar sources.

Aims. The Cygnus region in the northern sky is one of the richest in this type of sources that are potential counterparts to VHE sources. We surveyed the central $\sim 15 \mathrm{sq} \mathrm{deg}$ of the Cygnus constellation at the 325 and $610 \mathrm{MHz}$ bands with angular resolutions and sensitivities of $10^{\prime \prime}$ and $6^{\prime \prime}$, and 0.5 and $0.2 \mathrm{mJy} \mathrm{beam}^{-1}$, respectively.

Methods. The data were collected during $172 \mathrm{~h}$ in 2013-2017, using the Giant Metrewave Radio Telescope with $32 \mathrm{MHz}$ bandwidth, and were calibrated using the SPAM routines. The source extraction was carried out with the PyBDSF tool, followed by verification through visual inspection of every putative catalog candidate source in order to determine its reliability.

Results. In this first paper we present the catalog of sources, consisting of 1048 sources at $325 \mathrm{MHz}$ and 2796 sources at $610 \mathrm{MHz}$. By cross-matching the sources from both frequencies with the objects of the SIMBAD database, we found possible counterparts for 143 of them. Most of the sources from the 325-MHz catalog (993) were detected at the $610 \mathrm{MHz}$ band, and their spectral index $\alpha$ was computed adopting $S(v) \propto v^{\alpha}$. The maximum of the spectral index distribution is at $\alpha=-1$, which is characteristic of nonthermal emitters and might indicate an extragalactic population.
\end{abstract}

Key words. catalogs - radio continuum: general - open clusters and associations: individual: Cyg OB2 open clusters and associations: individual: Cyg OB8 - open clusters and associations: individual: Cyg OB9

\section{Introduction}

The first gamma-ray all-sky observations, obtained decades ago with the satellites COS-B (Hermsen et al. 1977, and references therein) and Compton (Hartman et al. 1999), disclosed numerous sources with no counterpart at other wavelengths. These are hereafter called unidentified gamma-ray sources, or UNIDS. Since then, a large number of multifrequency observations have been implemented to understand the nature of these sources (e.g., Paredes et al. 2008; Massaro et al. 2013). Despite significant improvement in the telescope capabilities in sensitivity and resolution, there still remain thousands of gamma-ray sources to be identified. For instance, the fourth catalog of the Fermi Large Area Telescope (The Fermi-LAT collaboration 2020, more than 5000 sources) listed about one-third of the detected sources without any counterpart at lower energies. The sources that were detected with ground-based telescopes, at $\mathrm{TeV}$ energies, also present problems in conclusive identification; in addition, the high uncertainty on their position precludes the correlation with

\footnotetext{
* Full Tables 3-5 are only available at the CDS via anonymous ftp to cdsarc.u-strasbg. fr (130.79.128.5) or via http://cdsarc. u-strasbg.fr/viz-bin/cat/J/A+A/642/A136
}

individual objects (see, e.g., the High Energy Stereoscopic System, H.E.S.S., source catalog ${ }^{1}$ and its identifications).

The identified gamma-ray sources are mostly active galactic nuclei, AGNs, and pulsars, supernova remnants, or high-mass Xray binaries (HMXBs). These objects emit at radio wavelengths and are generally stronger at low radio frequencies $(<1 \mathrm{GHz})$ as a result of the nature of the spectra of synchrotron radiation. In this part of the electromagnetic spectrum, major catalogs and surveys lack angular resolution or sensitivity to seek for singular counterparts of UNIDS (e.g., the National Radio Astronomy Observatory Very Large Array Sky Survey, NVSS, $\sim 45^{\prime \prime}$ and $1 \mathrm{mJy}$, or the Westerbork Northern Sky Survey, 54" and 3 mJy; Condon et al. 1998; Rengelink et al. 1997). Recently, other types of stellar sources have been proposed as possible gamma-ray emitters, and different scenarios were analyzed. In addition to the well-studied microquasars (Romero et al. 2003), colliding-wind binaries (Benaglia \& Romero 2003), Herbig Haro objects, young stellar objects (YSO), (Bosch-Ramon et al. 2010; Araudo et al. 2007; Rodríguez-Kamenetzky et al. 2019),

\footnotetext{
1 https://wWW.mpi-hd.mpg.de/hfm/HESS/pages/home/ sources/
} 
and stellar bow shocks (Benaglia et al. 2010; del Valle \& Pohl 2018; del Palacio et al. 2018) are capable of producing gammarays. A signature of high-energy emission is nonthermal radio emission because particles from the same population are likely to be involved in processes at both energy ranges, at the radio through synchrotron process, and at VHE emission through inverse-Compton scattering. Moreover, the determination of counterparts of gamma-ray sources through radio observations in star-forming regions will help to clarify the role of young stars and collective wind effects in the acceleration of galactic cosmic rays (e.g., Romero et al. 2008).

Various high-energy sources have been detected in the northern-sky Cygnus rift, a large region with star-formation activity that is one of the richest and most crowded in stellar objects in the Galaxy. Many thousand sources are cataloged in the literature in this region, and more than half are stars. The high absorption in the line of sight, however, prevents accurate mapping of the stellar population at the optical and IR ranges. Low-frequency (centimeter wavelengths) observations are the only way to probe nonthermal radio emission, and this emission travels practically unabsorbed; observing facilities that provide high angular resolution and sensitivity are crucial. In this line, the Giant Metrewave Radio Telescope (GMRT) is ideal for sampling the sky to search for emission of stellar sources: it operates between 150 and $1400 \mathrm{MHz}$, with baselines along $25 \mathrm{~km}$ that allow images with an angular resolution of a few arcseconds (Swarup et al. 1991).

We carried out a survey of the center of the Cygnus rift with the GMRT by means of continuum observations at two bands (325 MHz and $610 \mathrm{MHz}$ ) to investigate the nonthermal emission of various types of sources that lie in this rich field and are potential counterparts of UNIDS. With two frequencies, we were also able to obtain spectral information that might help to categorize certain classes and emission mechanisms on the basis of the spectral index. We present the source catalog at each band here, along with spectral index information when possible. In Sect. 2 we present the main characteristics of the Cygnus region and precedent studies at low-frequency radio continuum; in Sects. 3 and 4 we describe how the observations were carried out, and the processes attached to the data reduction to obtain the final images. Section 5 explains the data analysis we performed on the images and how the sources were extracted. Section 6 contains the findings related to spectral indices for the sources we detected at the two observing bands. In Sect. 7 we discuss the main properties of the catalog. Results of the search for counterparts are given in Sect. 8, and we conclude by mentioning related studies and prospects in the last section.

\section{Cygnus region and background of the radio observations}

The Cygnus rift is a large area at northern declination that is obscured by the dust of molecular clouds. It spans from $65^{\circ} \leq$ $l \leq 95^{\circ},-8^{\circ} \leq b \leq+8^{\circ}$ at a distance up to $2.5 \mathrm{kpc}$; see Reipurth et al. (2008) for a comprehensive review. As portrayed in Fig. 1 (Mahy et al. 2013), it encompasses nine OB associations and several bright open clusters, with signs of recent star formation. One of the youngest associations is Cyg OB2: it is also the richest association, with more than one hundred $\mathrm{O}$ stars and thousands of B stars, as reported by Knödlseder (2000). Next to OB2, Cyg OB8 and Cyg OB9 present hundreds of hot stars.

The main goal of the project was to relate nonthermal radio sources with stellar objects, that is, stars at different evolutionary stages: we circumscribed the region under study to the asso-

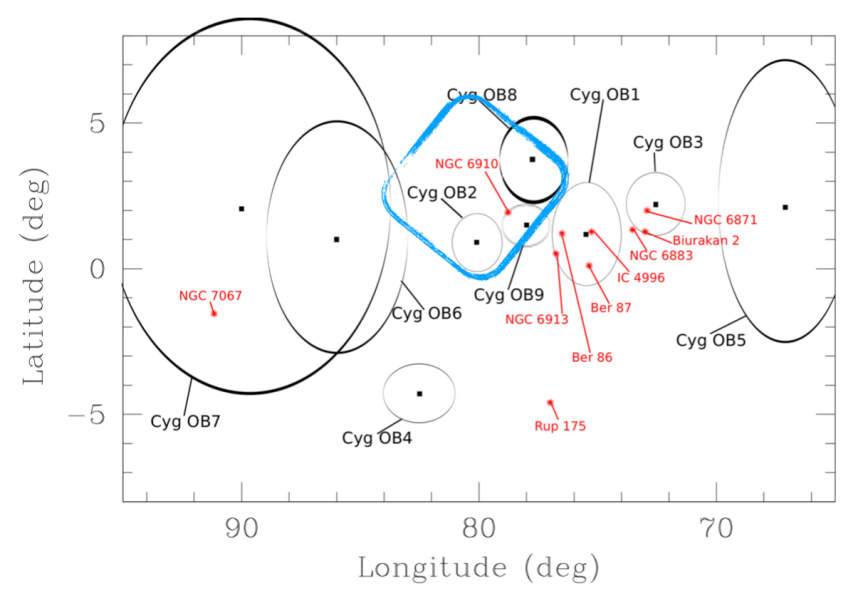

Fig. 1. Observed area of the Cygnus constellation marked with a blue contour box, over the stellar associations and bright clusters from Mahy et al. (2013).

ciations Cyg OB2, OB8 and OB9; its extension is displayed in Fig. 1 in Galactic coordinates, related to the Cygnus constellation. It covers $\sim 15 \mathrm{sqdeg}$. The associations Cyg OB8 and OB9 are not surveyed in full because they are adjacent to strong and/or large sources (like the cases of Cyg X-1 and Cyg A), which might introduce problems related to imaging of a highly dynamic range.

Below the Jansky threshold, the Cygnus area was observed as part of Galactic plane surveys with the Very Large Array ${ }^{2}$ by Garwood et al. (1988) at $1.4 \mathrm{GHz}$ continuum, at $b=0^{\circ}$, a resolution up to $4^{\prime \prime}$, and completely to a peak flux density of about $30 \mathrm{mJy}$. The results were complemented by those of Zoonematkermani et al. (1990) for $|b|<0.8^{\circ}$, which provided angular resolution and flux limit alike. With the Texas Interferometer, Douglas et al. (1996) imaged the area at the arcsecond scale above flux densities of 0.25-0.4 Jy. Taylor et al. (1996) carried out Westerbork Synthesis Radio Telescope observations along the Galactic plane and for $|b|<1.6^{\circ}$, at an angular resolution of $\sim 1^{\prime}$. They detected sources brighter than $10 \mathrm{mJy} \mathrm{beam}^{-1}$.

In particular, Setia Gunawan et al. (2003) published the Westerbork Synthesis Radio Telescope 1400 and $325 \mathrm{MHz}$ continuum survey of Cyg OB2, which attained angular resolutions of $13^{\prime \prime}$ and $55^{\prime \prime}$ and $5 \sigma$ flux density limits of $\sim 2 \mathrm{mJy}$ and $\sim 10$ $15 \mathrm{mJy}$, respectively. In an observed area of $2^{\circ} \times 2^{\circ}$, the authors detected 210 discrete sources, 98 of them at both frequencies. They also detected 28 resolved sources.

The observations presented here were performed at two bands with the GMRT, centered at 325 and $610 \mathrm{MHz}$. This allowed us to map the continuum radio emission at arcsecond resolution and below the mJy sensitivity level. Some information about the observations has been given in Ishwara-Chandra et al. (2019).

\section{Observations}

The observed region marked in Fig. 1 is displayed in equatorial coordinates in Fig. 2. The half-power beam widths of the GMRT fields of view (FoVs) are $81 \pm 4^{\prime}$ at $325 \mathrm{MHz}$ and $43 \pm 3^{\prime}$ at $610 \mathrm{MHz}^{3}$.

To cover the desired observing area, we needed to point at 5 FoVs of $325 \mathrm{MHz}$ and $47 \mathrm{FoVs}$ of $610 \mathrm{MHz}$. Some observations

\footnotetext{
2 https://science.nrao.edu/facilities/vla

3 GMRT Observer's Manual; http://www.ncra.tifr.res.in/ ncra/gmrt/gmrt-users/observing-help/manual_7jul15.pdf
} 


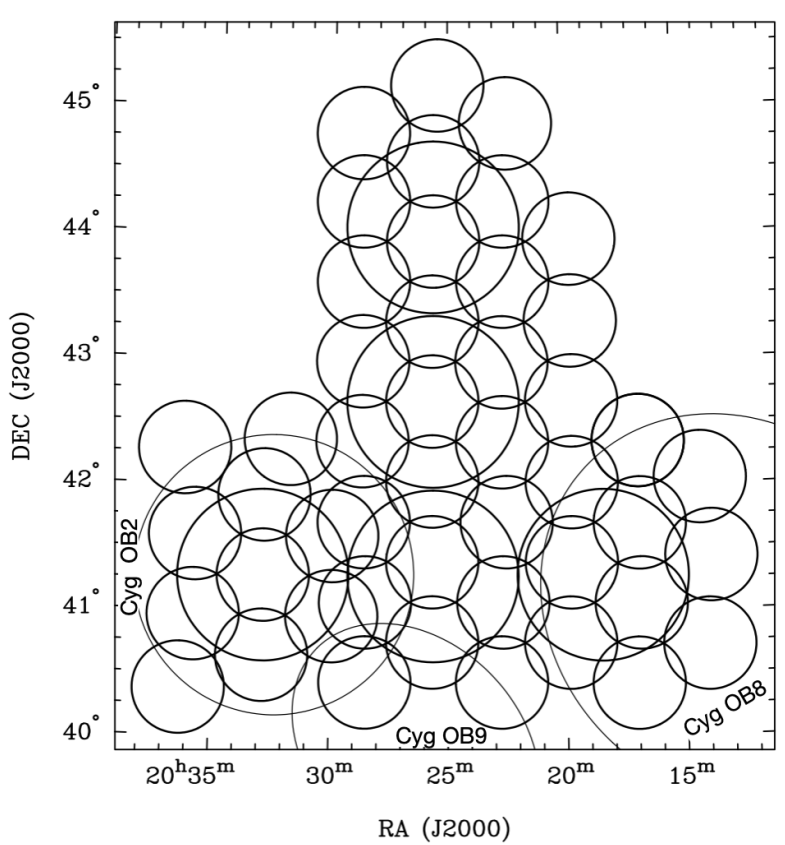

Fig. 2. Disposition of the pointings at $325 \mathrm{MHz}$ (larger circles) and $610 \mathrm{MHz}$ (smaller circles), showing the observed FoV half-power beam widths. The extent of the stellar associations Cyg OB2, OB8, and OB9 (Uyanıker et al. 2001) is shown using thinner lines.

Table 1. Observing campaigns basic information.

\begin{tabular}{cccc}
\hline \hline$\#$ & Campaing ID & Time (h) & Obs. dates \\
\hline 1 & 25_026 & 12 & 2013 \\
2 & 27_036 & 40 & $2014-2015$ \\
3 & 28_081 & 40 & 2015 \\
4 & 30_027 & 60 & $2016-2017$ \\
\hline
\end{tabular}

consisted of bad data, so that we repeated them with new observations $(20 \mathrm{~h})$. The layout of the pointings was chosen to yield a uniform noise while minimizing the number of them (i.e., the observing time). Figure 2 shows the disposition of the pointings and the FoVs at both observing bands. The project was divided into four observing campaigns, scheduled from November 2013 to September 2017. Table 1 lists the GMRT campaign ID, the allocated time, and the year(s) of completion.

Details of the targeted areas and observing parameters are given in Table 2: the name of the FoV, the corresponding campaign ID, the exact observation date, the position of the pointing center, the time on the FoVs (t.o.s.), the band, and the calibrators, ordered by band and by right ascension. The observations were carried out using the total intensity mode and a bandwidth of 32 $\mathrm{MHz}$ and 256 spectral channels to minimize the effect of bandwidth smearing. Flux calibrators were observed at the start and end of the each run for flux and bandpass calibration. A phase calibrator was observed for $5 \mathrm{~min}$ after a scan of $30 \mathrm{~min}$ on the target to calibrate the phases and any slow variations of the telescope gain.

\section{Data reduction}

The five pointings at $325 \mathrm{MHz}$ were processed uniformly, using the SPAM routines (Intema 2014), which is a python package based on the Astronomical Image Processing System (AIPS) for nearly automatic analysis of GMRT data below $1 \mathrm{GHz}$. Bad data and RFI were initially flagged at the full spectral resolution of 256 channels. The flux was calibrated using the primary calibrators 3C286, 3C48, and 3C147 and the scale by Scaife \& Heald (2012) for low radio frequencies. The SPAM pipeline then converted the precalibrated visibility data to a final image, which includes several rounds of self-calibration and flagging iteratively, and wide-field imaging to correct for noncoplanarity. In the self-calibration, ionospheric phase corrections were computed for several directions within the FoV for directiondependent corrections on the integration timescales. The selfcalibration procedure was followed using default parameters of SPAM, with initial cycles in phase with long intervals and a last run with the solution interval of the visibility integration time; in our case, 16.9 seconds. Toward the end of the loop, one round of amplitude and phase self-calibration was carried out. During imaging, a moderately uniform weighting scheme (robust $=-1$ in AIPS) was used, but no multiscale cleaning options were incorporated. Primary-beam corrections were applied using the GMRT specific parameters (GMRT Observer's Manual).

Because the target is in the Galactic plane, the $T_{\text {sys }}$ correction for excess background was applied using the $408 \mathrm{MHz}$ allsky map (Haslam et al. 1982) during the calibration as part of the pipeline. The correction factor varied from 1.7 to 3.6 , with the highest correction factor near the Galactic plane and a lower correction factor away from the plane. The FoVs were combined in a mosaic with weights as inverse of the variance.

The data analysis of the $47 \mathrm{FoVs}$ at $610 \mathrm{MHz}$ was also carried out using the SPAM pipeline, similar to the $325 \mathrm{MHz}$ data. The $T_{\text {sys }}$ correction for the excess background at 610 $\mathrm{MHz}$ ranged from 1.22 to 1.76 . Fields of view FoV610.21 and FoV610.30 were noisier than the rest, probably because of strong extended emission from the Galactic plane and bright sources in the fields.

The final mosaics presented an average rms of $0.5 \mathrm{mJy} \mathrm{beam}^{-1}$ and $0.2 \mathrm{mJy}$ beam $^{-1}$ at 325 and $610 \mathrm{MHz}$, respectively, although locally, the values strongly depend mainly on the extended and/or diffuse emission. The synthesized beams attained were $10^{\prime \prime} \times 10^{\prime \prime}$, and $6^{\prime \prime} \times 6^{\prime \prime}$, and the mosaic image sizes were $(6487 \times 6573)$, and $(12580 \times 13837)$ pixels, respectively. The final images are presented in Figs. 3 and 4 .

A series of factors affect the accuracy of low-frequency radio flux scales in different ways. This in turn affects the uncertainties. In their observations with the GMRT, Chandra et al. (2004), for instance, discussed a flux-scale uncertainty at 325 and 610 $\mathrm{MHz}$ of a few percent. In addition, when the target fields lie in the Galactic plane, as the field we present, the fact that $T_{\text {sys }}$ can be significantly higher because the sky temperature is higher than toward the calibrator imposes an additional correction factor; although the SPAM pipeline estimates this factor, it is based in some assumptions and extrapolations that may introduce some inaccuracy. We used different flux calibrators, the primary-beam model is not perfect, and mosaicking different pointings into the final images we used for source extraction can all affect the flux scale. When all of this is taken into account, a very conservative approach is to adopt flux density uncertainties of $10 \%$.

\section{Source extraction}

When the mosaics at the two observing bands were built, we searched for errors in the astrometry, first, by taking point sources in the entire two images into consideration. We did not find significant errors at the smaller pixel scale, which means that 
Table 2. Observing runs and fields of view information.

\begin{tabular}{|c|c|c|c|c|c|c|c|}
\hline \multirow{2}{*}{$\begin{array}{l}\text { Field of view } \\
\text { name }\end{array}$} & \multirow{2}{*}{$\begin{array}{c}\text { Campaign } \\
\text { ID }\end{array}$} & \multirow{2}{*}{$\begin{array}{c}\text { Observing } \\
\text { dates }\end{array}$} & \multicolumn{2}{|c|}{ Pointing center (J2000) } & \multirow{2}{*}{$\begin{array}{l}\text { t.o.s. } \\
(\mathrm{min})\end{array}$} & \multirow{2}{*}{$\begin{array}{c}\text { Band } \\
(\mathrm{MHz})\end{array}$} & \multirow[t]{2}{*}{ Calibrators } \\
\hline & & & $\mathrm{RA}(\mathrm{h}, \mathrm{m}, \mathrm{s})$ & Dec (deg,,',') & & & \\
\hline FoV325.1 & 27_036 & $7 / 2,26 / 9 / 15$ & 201826 & 411650 & 488 & 325 & $3 C 48,2052+365$ \\
\hline FoV325.2 & 27_036 & $26 / 10 / 14$ & 202538 & 411650 & 304 & 325 & $3 C 48,2052+365$ \\
\hline FoV325.3 & 27_036 & $27 / 10 / 14$ & 202538 & 423950 & 296 & 325 & $3 \mathrm{C} 147,2052+365$ \\
\hline FoV325.4 & 27_036 & $6 / 2 / 15$ & 202538 & 440250 & 283 & 325 & $3 \mathrm{C} 48,2052+365$ \\
\hline FoV325.5 & 25_026 & $4 / 11 / 13$ & 203250 & 411650 & 523 & 325 & $3 \mathrm{C} 48,2038+513$ \\
\hline$\overline{\text { FoV610.1 }}$ & 28_081 & $18 / 6 / 15$ & 201350 & 412115 & 79 & 610 & $3 C 48,2052+365$ \\
\hline FoV610.2 & 28_081 & $18 / 6 / 15$ & 201360 & 404310 & 79 & 610 & $3 \mathrm{C} 48,2052+365$ \\
\hline FoV610.3 & 30_027 & $11,21 / 8 / 16$ & 201413 & 420230 & 118 & 610 & $3 \mathrm{C} 48,3 \mathrm{C} 286,2052+365$ \\
\hline FoV610.4 & 28_081 & $18 / 6 / 15$ & 201650 & 410315 & 130 & 610 & $3 \mathrm{C} 48,2052+365$ \\
\hline FoV610.5 & 28_081 & $25 / 7 / 15$ & 201650 & 414120 & 119 & 610 & $3 C 48,2052+365$ \\
\hline FoV610.6 & 30_027 & $2 / 9 / 17$ & 201650 & 422030 & 69 & 610 & $3 C 48,2052+365$ \\
\hline FoV610.7 & 28_081 & $25 / 7 / 15$ & 201700 & 402505 & 71 & 610 & $3 C 48,2052+365$ \\
\hline FoV610.8 & 30_027 & $17 / 7,8 / 8 / 16$ & 201940 & 424001 & 147 & 610 & $3 C 48,2052+365$ \\
\hline FoV610.9 & 30_027 & $17 / 7,8 / 8 / 16$ & 201940 & 431801 & 179 & 610 & $3 C 48,2052+365$ \\
\hline FoV610.10 & 30_027 & $17 / 7 / 16$ & 201940 & 435656 & 78 & 610 & $3 C 48,2052+365$ \\
\hline FoV610.11 & 30_027 & $1 / 7 / 16$ & 201942 & 420108 & 94 & 610 & $3 \mathrm{C} 286,2052+365$ \\
\hline FoV610.12 & 28_081 & $25 / 7 / 15$ & 201945 & 412260 & 119 & 610 & $3 C 48,2052+365$ \\
\hline FoV610.13 & 28_081 & $15 / 8 / 15$ & 201950 & 404450 & 119 & 610 & $3 C 48,2052+365$ \\
\hline FoV610.14 & 30_027 & $15 / 7 / 16$ & 202225 & 445209 & 64 & 610 & $3 C 48,2052+365$ \\
\hline FoV610.15 & 28_081 & $16 / 8 / 15$ & 202230 & 414230 & 119 & 610 & $3 C 48,2052+365$ \\
\hline FoV610.16 & 30_027 & $2 / 9 / 17$ & 202234 & 441501 & 59 & 610 & $3 C 48,2052+365$ \\
\hline FoV610.17 & 30_027 & $2 / 9 / 17$ & 202236 & 433653 & 59 & 610 & $3 C 48,2052+365$ \\
\hline FoV610.18 & 28_081 & $15 / 8 / 15$ & 202240 & 410420 & 107 & 610 & $3 \mathrm{C} 48,2052+365$ \\
\hline FoV610.19 & 30_027 & $30 / 6,23 / 7 / 16$ & 202240 & 422030 & 168 & 610 & $3 C 48,3 C 286,2052+365$ \\
\hline FoV610.20 & 30_027 & $30 / 6,23 / 7 / 16$ & 202240 & 425830 & 231 & 610 & $3 \mathrm{C} 48,3 \mathrm{C} 286,2052+365$ \\
\hline FoV610.21 & 28_081 & $15 / 8 / 15$ & 202245 & 402615 & 62 & 610 & $3 C 48,2052+365$ \\
\hline FoV610.22 & 30_027 & $15 / 7 / 16$ & 202527 & 451021 & 71 & 610 & $3 C 48,2052+365$ \\
\hline FoV610.23 & 30_027 & $2 / 9 / 17$ & 202538 & 435606 & 76 & 610 & $3 C 48,2052+365$ \\
\hline FoV610.24 & 30_027 & $2 / 9 / 17$ & 202538 & 443414 & 70 & 610 & $3 C 48,2052+365$ \\
\hline FoV610.25 & 28_081 & $16 / 8 / 15$ & 202540 & 404525 & 119 & 610 & $3 \mathrm{C} 48,2052+365$ \\
\hline FoV610.26 & 28_081 & $17 / 8 / 15$ & 202540 & 412335 & 142 & 610 & $3 C 48,2052+365$ \\
\hline FoV610.27 & 30_027 & $14 / 7 / 16$ & 202540 & 420160 & 89 & 610 & $3 \mathrm{C} 48,2052+365$ \\
\hline FoV610.28 & 30_027 & $30 / 6,23 / 7 / 16$ & 202540 & 424000 & 168 & 610 & $3 \mathrm{C} 48,3 \mathrm{C} 286,2052+365$ \\
\hline FoV610.29 & 30_027 & $1,2,24 / 7 / 16$ & 202540 & 431800 & 243 & 610 & $3 \mathrm{C} 48,3 \mathrm{C} 286,2052+365$ \\
\hline FoV610.30 & 28_081 & $17 / 8 / 15$ & 202830 & 402615 & 79 & 610 & $3 \mathrm{C} 48,2052+365$ \\
\hline FoV610.31 & 28_081 & $16 / 8 / 15$ & 202830 & 410420 & 71 & 610 & $3 C 48,2052+365$ \\
\hline FoV610.32 & 28_081 & $17 / 8 / 15$ & 202835 & 414230 & 79 & 610 & $3 \mathrm{C} 48,2052+365$ \\
\hline FoV610.33 & 30_027 & $1,2,24 / 7 / 16$ & 202840 & 422100 & 232 & 610 & $3 \mathrm{C} 48,3 \mathrm{C} 286,2052+365$ \\
\hline FoV610.34 & 30_027 & $1,2,24 / 7 / 16$ & 202840 & 425900 & 230 & 610 & $3 C 48,3 C 286,2052+365$ \\
\hline FoV610.35 & 30_027 & $1 / 7 / 16$ & 202840 & 433653 & 94 & 610 & $3 C 286,2052+365$ \\
\hline FoV610.36 & 30_027 & $1 / 7 / 16$ & 202842 & 441501 & 94 & 610 & $3 \mathrm{C} 286,2052+365$ \\
\hline FoV610.37 & 30_027 & $15 / 7 / 16$ & 202844 & 444732 & 71 & 610 & $3 \mathrm{C} 48,2052+365$ \\
\hline FoV610.38 & 27_036 & $28,29 / 11 / 14$ & 202955 & 405738 & 120 & 610 & $3 C 48,2052+365$ \\
\hline FoV610.39 & 27_036 & $28,29 / 11 / 14$ & 202955 & 413545 & 120 & 610 & $3 C 48,2052+365$ \\
\hline FoV610.40 & 30_027 & $17 / 7,8 / 8 / 16$ & 203145 & 422139 & 145 & 610 & $3 C 48,2052+365$ \\
\hline FoV610.41 & 27_036 & $28,29 / 11 / 14$ & 203250 & 403842 & 120 & 610 & $3 C 48,2052+365$ \\
\hline FoV610.42 & 30_027 & $14 / 7,8 / 8 / 16$ & 203250 & 411650 & 165 & 610 & $3 C 48,2052+365$ \\
\hline FoV610.43 & 27_036 & $28,29 / 11 / 14$ & 203250 & 415458 & 120 & 610 & $3 C 48,2052+365$ \\
\hline FoV610.44 & 27_036 & $28,29 / 11 / 14$ & 203545 & 405738 & 105 & 610 & $3 C 48,2052+365$ \\
\hline FoV610.45 & 27_036 & $28,29 / 11 / 14$ & 203545 & 413545 & 120 & 610 & $3 \mathrm{C} 48,2052+365$ \\
\hline FoV610.46 & 30_027 & $8,11,21 / 8 / 16$ & 203617 & 402230 & 177 & 610 & $3 \mathrm{C} 48,3 \mathrm{C} 286,2052+365$ \\
\hline FoV610.47 & 30_027 & $11,21 / 8 / 16$ & 203617 & 421630 & 118 & 610 & $3 \mathrm{C} 48,3 \mathrm{C} 286,2052+365$ \\
\hline
\end{tabular}

the accuracy was better than 1.5 arcsec. We also searched the $610 \mathrm{MHz}$ image and point sources with well-determined optical positions, that is, Wolf-Rayet and O-type stars. For the cases with radio emission at or near the position of these stars (13 in total), the differences in coordinates were in the range $0.1-3.1^{\prime \prime}$, and the standard deviation was 1.34" (see Benaglia et al. 2020, for a study of the massive early-type stars detected in the current databases). 


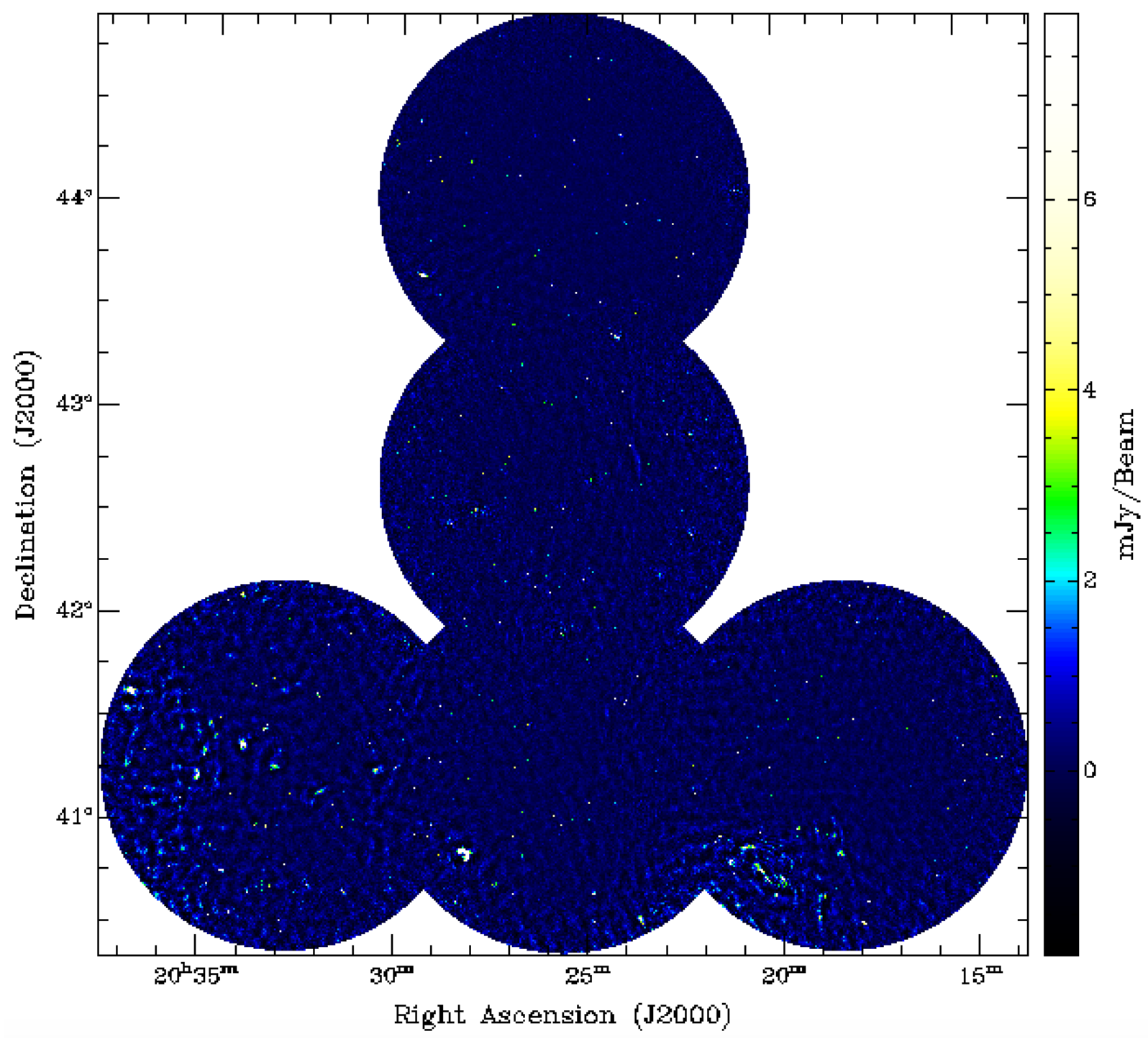

Fig. 3. GMRT $325 \mathrm{MHz}$ continuum image of the observed field. The synthesized beam is $10^{\prime \prime} \times 10^{\prime \prime}$, and the average rms is $0.5 \mathrm{mJy}$ beam ${ }^{-1}$. The full range of the flux density values is $-9.2,+812.5 \mathrm{mJy} \mathrm{beam}^{-1}$. The interval shown is $(-2,+8) \mathrm{mJy}_{\text {beam }}{ }^{-1}$ to outline the weaker features.

To survey the emission in the 325 and $610 \mathrm{MHz}$ images, we applied the Python blob detector and source finder $\left(\mathrm{PyBDSF}^{4}\right)$. The tool can be used to find islands of emission in radio interferometry images, decompose them into Gaussian functions, and finally gather them into individual source fits. We chose a signal-to-noise ratio of 7 as the lower limit for a source or fit to be accepted, and proceeded in the same way as Benaglia et al. (2019), where this proved successful. A similar detection threshold was used for the CORNISH catalog (Purcell et al. 2013). The routine includes the determination of the rms in the image and the production of a corresponding rms image.

The running of PyBDSF over the 325 and $610 \mathrm{MHz}$ image mosaics resulted in 1230 and 3023 sources, respectively. After a thorough visual inspection of each source, we kept 1048 (85.2\%) sources from the $325 \mathrm{MHz}$ image and 2796 (92.5\%) sources from the $610 \mathrm{MHz}$ image.

The group of accepted entries consisted of discrete (unresolved) objects that were represented by one fitted source of the size of the synthesized beam, and of resolved objects. Some resolved objects were represented by a fitted source that was

\footnotetext{
4 http://www.astron.nl/citt/pybdsf/
}

larger than the synthesized beam, while others were described by a combination of fitted sources.

We rejected fits to filaments and (part of) diffuse emission (3.5\% at $325 \mathrm{MHz}$ and $1.5 \%$ at $610 \mathrm{MHz}$, see Fig. 5a) and the fit combinations that corresponded to strong and/or large objects with ill representations $(1.7 \%$ at $325 \mathrm{MHz}$ and $2.0 \%$ at $610 \mathrm{MHz}$; Fig. 5b). We also discarded either objects with reduction artifacts that precluded a proper fit (including end-of-field objects: $2.5 \%$ at $325 \mathrm{MHz}$ and $8.2 \%$ at $610 \mathrm{MHz}$, Fig. 5c) and fitted sources similar to surrounding noise $(1.5 \%$ at $325 \mathrm{MHz}$ and $1.3 \%$ at $610 \mathrm{MHz}$, see Fig. 5d).

Overall, the $610 \mathrm{MHz}$ emission was better imaged by the SPAM pipelines than the emission at the lower frequency band. The $325 \mathrm{MHz}$ mosaic presented a higher percentage of extended emission-fitting problems. The largest detectable structure is $32^{\prime}$ at the $325 \mathrm{MHz}$ band and $17^{\prime}$ at the $610 \mathrm{MHz}$ band (GMRT User's Manual): the data presented here are biased against structures that are larger than that. The selection of the robust weighting of $-1, \mathrm{a}$ compromise between high angular resolution and signal-to-noise ratio, outlined discrete sources over diffuse emission.

The attained rms at the two bands, in addition to the intrinsic values contributed by the stellar sources under observation, are 


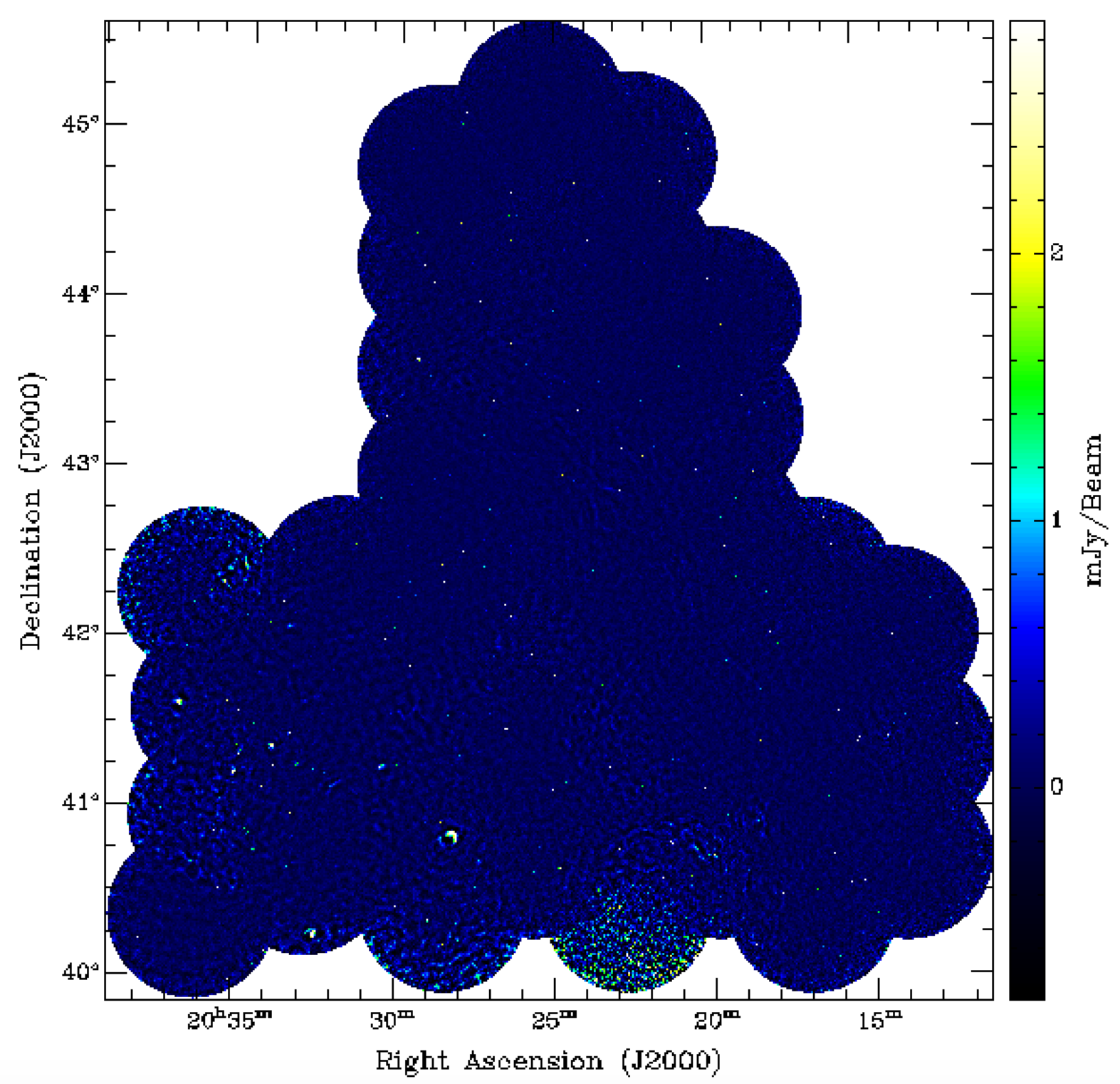

Fig. 4. GMRT $610 \mathrm{MHz}$ continuum image of the observed field. The synthesized beam is $6^{\prime \prime} \times 6^{\prime \prime}$, and the average rms is $0.2 \mathrm{mJy}$ beam ${ }^{-1}$. The full range of the flux density values is $-6.3,+928.3 \mathrm{mJy}_{\text {beam }}{ }^{-1}$. The interval shown is $(-0.8,+2.9) \mathrm{mJy}_{\text {beam }}{ }^{-1}$ to outline the weaker features.

indeed a function of the time-on-source (here, time-on-fields). To determine the completeness of the sources detected above $7 \sigma(7 \mathrm{rms})$, we also ran the PyBDSF routines using a detection threshold of $5 \sigma$. We found 1721 fitted sources at $325 \mathrm{MHz}$ and 5015 at $610 \mathrm{MHz}$, which is well above the numbers found for the first run. When we visually inspected several faint sources, they appeared like noise peaks, which prompted us to use $7 \sigma$, with which we found significantly fewer spurious sources. The incompleteness of our catalog is mainly quantified by the reasons given above regarding the fits.

The final lists of the accepted objects are reunited in our catalog. The cataloged sources are given in Tables 3 and 4, named consecutively (Col. 1) by increasing right ascension; only sample items are shown. We tagged the sources with the label "BIC" followed by the observing frequency in $\mathrm{MHz}$, and then a correlative number, based on their order. For each source, we list the coordinates RA, Dec (J2000) of the fit (Cols. 2 and 3), the integrated flux (Col. 4), the peak flux (Col. 5) and the fitted major axis, minor axis, and position angle $\left(\theta_{1}, \theta_{2}\right.$, and $P A$, Cols. 6-8), which represent the source size and orientation after deconvolu- tion, all with their corresponding errors as reported by PyBDSF. The full Tables 3 and 4 are provided at the CDS.

\section{Determining spectral indices}

The spectral index $\alpha$ of a source is a key parameter in the determination of its nature. When the flux densities at frequency bands centered at $v_{1}$ and $v_{2}$ are $S_{1}$ and $S_{2}$, and $S_{v} \propto v^{\alpha}$, $\alpha=\log \left(S_{1} / S_{2}\right) / \log \left(v_{1} / v_{2}\right)$. For the observations we processed, $v_{1}=325 \mathrm{MHz}$ and $v_{2}=610 \mathrm{MHz}$, and $\alpha$ can be derived when a source was detected at both bands.

In the process of obtaining spectral index information, we verified whether the sources we detected at one of the observing bands were positionally coincident with one or more sources detected at the other band. To do this, we determined for each $325 \mathrm{MHz}$ source ellipse whether it overlapped one or more of the $610 \mathrm{MHz}$ source ellipses. When this was the case, we classified the overlapping as partial when the ellipse at $325 \mathrm{MHz}$ partially overlapped the ellipse at $610 \mathrm{MHz}$, or as full when the $610 \mathrm{MHz}$ 
P. Benaglia et al.: Cygnus survey with the Giant Metrewave Radio Telescope at 325 and $610 \mathrm{MHz}$ : the catalog

Table 3. Detected sources at $325 \mathrm{MHz}$ above the $7 \sigma$ level (first entries).

\begin{tabular}{lccccccc}
\hline \hline ID & $\begin{array}{c}\text { RA }_{\text {J2000 }} \\
(\mathrm{h}, \mathrm{m}, \mathrm{s})\end{array}$ & $\begin{array}{c}\text { Dec }_{\text {J2000 }} \\
\left(\mathrm{deg},{ }^{\prime \prime}{ }^{\prime}\right)\end{array}$ & $\begin{array}{c}\text { Total flux } \\
(\mathrm{mJy})\end{array}$ & $\begin{array}{c}\text { Peak flux } \\
(\mathrm{mJy} / \mathrm{beam})\end{array}$ & $\begin{array}{c}\theta_{1} \\
\left({ }^{\prime \prime}\right)\end{array}$ & $\begin{array}{c}\theta_{2} \\
\left({ }^{\prime \prime}\right)\end{array}$ & $\begin{array}{c}P A \\
\left({ }^{\circ}\right)\end{array}$ \\
\hline BIC325-0001 & $20: 13: 54.55 \pm 0.049$ & $41: 32: 32.01 \pm 0.88$ & $4.7 \pm 1.00$ & $2.8 \pm 0.40$ & $13.9 \pm 2.10$ & $13.9 \pm 2.10$ & $16.0 \pm 46.54$ \\
BIC325-0002 & $20: 13: 56.45 \pm 0.025$ & $41: 33: 06.07 \pm 0.30$ & $16.7 \pm 1.19$ & $7.7 \pm 0.39$ & $17.2 \pm 0.97$ & $17.2 \pm 0.97$ & $56.4 \pm 6.23$ \\
BIC325-0003 & $20: 14: 04.78 \pm 0.024$ & $40: 56: 14.47 \pm 0.27$ & $5.1 \pm 0.63$ & $5.0 \pm 0.36$ & $10.8 \pm 0.83$ & $10.8 \pm 0.83$ & $93.3 \pm 21.93$ \\
BIC325-0004 & $20: 14: 07.82 \pm 0.037$ & $41: 02: 47.35 \pm 0.61$ & $3.4 \pm 0.69$ & $2.8 \pm 0.36$ & $11.2 \pm 1.44$ & $11.2 \pm 1.44$ & $7.9 \pm 87.95$ \\
BIC325-0005 & $20: 14: 17.56 \pm 0.003$ & $41: 29: 24.16 \pm 0.03$ & $80.9 \pm 0.83$ & $58.9 \pm 0.38$ & $12.9 \pm 0.09$ & $12.9 \pm 0.09$ & $77.2 \pm 179.64$ \\
BIC325-0006 & $20: 14: 18.62 \pm 0.007$ & $41: 18: 10.88 \pm 0.07$ & $43.3 \pm 0.97$ & $25.0 \pm 0.38$ & $14.5 \pm 0.24$ & $14.5 \pm 0.24$ & $74.8 \pm 1.70$ \\
BIC325-0007 & $20: 14: 19.17 \pm 0.003$ & $41: 29: 36.95 \pm 0.04$ & $54.7 \pm 0.79$ & $42.2 \pm 0.38$ & $12.0 \pm 0.11$ & $12.0 \pm 0.11$ & $73.0 \pm 1.59$ \\
BIC325-0008 & $20: 14: 24.35 \pm 0.012$ & $41: 41: 32.07 \pm 0.36$ & $24.3 \pm 1.48$ & $10.4 \pm 0.38$ & $18.5 \pm 0.91$ & $18.5 \pm 0.91$ & $110.5 \pm 3.29$ \\
BIC325-0009 & $20: 14: 27.13 \pm 0.036$ & $41: 17: 52.59 \pm 0.36$ & $6.5 \pm 0.85$ & $4.4 \pm 0.37$ & $13.7 \pm 1.28$ & $13.7 \pm 1.28$ & $76.4 \pm 14.16$ \\
BIC325-0010 & $20: 14: 29.42 \pm 0.014$ & $41: 41: 32.46 \pm 0.13$ & $24.1 \pm 1.04$ & $14.0 \pm 0.41$ & $15.4 \pm 0.51$ & $15.4 \pm 0.51$ & $81.4 \pm 2.61$ \\
\hline
\end{tabular}

Notes. $\theta_{1}, \theta_{2}$, and $P A$ represent the elliptic source size and orientation, and correspond to the major axis, the minor axis, and the position angle of the fit by the PyBDSM routines. The full table with 1048 records is available at the CDS.

Table 4. Detected sources at $610 \mathrm{MHz}$ above the $7 \sigma$ level (first entries).

\begin{tabular}{lccccccc}
\hline \hline ID & $\begin{array}{c}\text { RA }_{\mathrm{J} 2000} \\
(\mathrm{~h}, \mathrm{~m}, \mathrm{~s})\end{array}$ & $\begin{array}{c}\text { Dec } \mathrm{J2000} \\
\left(\mathrm{deg},{ }^{\prime},{ }^{\prime \prime}\right)\end{array}$ & $\begin{array}{c}\text { Total flux } \\
(\mathrm{mJy})\end{array}$ & $\begin{array}{c}\text { Peak flux } \\
(\mathrm{mJy} / \mathrm{beam})\end{array}$ & $\begin{array}{c}\theta_{1} \\
\left(^{\prime \prime}\right)\end{array}$ & $\begin{array}{c}\theta_{2} \\
\left(^{\prime \prime}\right)\end{array}$ & $\begin{array}{c}P A \\
\left({ }^{\circ}\right)\end{array}$ \\
\hline BIC610-0001 & $20: 11: 32.02 \pm 0.009$ & $40: 53: 10.84 \pm 0.10$ & $14.5 \pm 0.69$ & $7.8 \pm 0.26$ & $9.3 \pm 0.34$ & $9.3 \pm 0.34$ & $63.3 \pm 3.83$ \\
BIC610-0002 & $20: 11: 33.03 \pm 0.001$ & $40: 53: 18.92 \pm 0.02$ & $63.8 \pm 0.75$ & $42.0 \pm 0.24$ & $7.9 \pm 0.06$ & $7.9 \pm 0.06$ & $60.6 \pm 1.35$ \\
BIC610-0003 & $20: 11: 34.94 \pm 0.009$ & $41: 31: 49.27 \pm 0.09$ & $12.5 \pm 0.63$ & $7.9 \pm 0.26$ & $8.8 \pm 0.33$ & $8.8 \pm 0.33$ & $112.7 \pm 2.90$ \\
BIC610-0004 & $20: 11: 48.15 \pm 0.019$ & $40: 51: 32.52 \pm 0.25$ & $2.3 \pm 0.40$ & $2.3 \pm 0.23$ & $6.6 \pm 0.73$ & $6.6 \pm 0.73$ & $53.2 \pm 23.77$ \\
BIC610-0005 & $20: 11: 53.58 \pm 0.007$ & $40: 48: 39.49 \pm 0.11$ & $8.6 \pm 0.50$ & $6.4 \pm 0.24$ & $7.4 \pm 0.29$ & $7.4 \pm 0.29$ & $128.0 \pm 11.67$ \\
BIC610-0006 & $20: 11: 55.45 \pm 0.008$ & $42: 13: 40.96 \pm 0.11$ & $4.3 \pm 0.33$ & $4.1 \pm 0.19$ & $6.3 \pm 0.30$ & $6.3 \pm 0.30$ & $106.9 \pm 27.75$ \\
BIC610-0007 & $20: 11: 56.63 \pm 0.014$ & $42: 13: 37.89 \pm 0.24$ & $2.4 \pm 0.34$ & $2.2 \pm 0.19$ & $6.7 \pm 0.60$ & $6.7 \pm 0.60$ & $142.6 \pm 23.84$ \\
BIC610-0008 & $20: 11: 58.89 \pm 0.009$ & $41: 47: 49.24 \pm 0.18$ & $2.0 \pm 0.27$ & $2.6 \pm 0.18$ & $5.6 \pm 0.42$ & $5.6 \pm 0.42$ & $1.1 \pm 21.54$ \\
BIC610-0009 & $20: 11: 59.38 \pm 0.007$ & $40: 28: 25.33 \pm 0.09$ & $7.6 \pm 0.44$ & $6.7 \pm 0.23$ & $6.8 \pm 0.25$ & $6.8 \pm 0.25$ & $57.4 \pm 11.20$ \\
BIC610-0010 & $20: 12: 00.79 \pm 0.018$ & $42: 02: 34.40 \pm 0.28$ & $2.1 \pm 0.34$ & $1.8 \pm 0.17$ & $6.7 \pm 0.69$ & $6.7 \pm 0.69$ & $134.7 \pm 70.97$ \\
\hline
\end{tabular}

Notes. $\theta_{1}, \theta_{2}$, and $P A$ represent the elliptic source size and orientation, and correspond to the major axis, the minor axis, and the position angle of the fit by the PyBDSM routines. The full table with 2796 records is available at the CDS.

Table 5. Sources detected at both frequency bands ( 325 and $610 \mathrm{MHz})$ and spectral index information (first entries).

\begin{tabular}{|c|c|c|c|}
\hline ID at $325 \mathrm{MHz}$ & $\begin{array}{l}\mathrm{RA}, \mathrm{Dec}_{\mathrm{J} 2000} \\
(\mathrm{hms}, \mathrm{dms})\end{array}$ & ID at $610 \mathrm{MHz}$ & $\alpha_{610 \mathrm{MHz}}^{325 \mathrm{MHz}}$ \\
\hline BIC325-0002 & $20: 13: 56.45,41: 33: 06.07$ & BIC610-0104, -0105 & $-0.4 \pm 0.26$ \\
\hline BIC325-0003 & $20: 14: 04.78,40: 56: 14.47$ & BIC610-0109 & $-0.7 \pm 0.32$ \\
\hline BIC325-0004 & $20: 14: 07.82,41: 02: 47.35$ & BIC610-0112 & $-1.4 \pm 0.48$ \\
\hline BIC325-0005 & $20: 14: 17.56,41: 29: 24.16$ & BIC610-0123 & $-1.1 \pm 0.23$ \\
\hline BIC325-0006 & $20: 14: 18.62,41: 18: 10.88$ & BIC610-0124 & $-1.0 \pm 0.23$ \\
\hline BIC325-0007 & $20: 14: 19.17,41: 29: 36.95$ & BIC610-0126 & $-0.9 \pm 0.23$ \\
\hline BIC325-0008 & $20: 14: 24.35,41: 41: 32.07$ & BIC610-0133, -0134, -0131 & $-0.8 \pm 0.25$ \\
\hline BIC325-0009 & $20: 14: 27.13,41: 17: 52.59$ & BIC610-0140 & $-1.2 \pm 0.34$ \\
\hline BIC325-0010 & $20: 14: 29.42,41: 41: 32.46$ & BIC610-0146 & $-1.3 \pm 0.24$ \\
\hline BIC325-0012 & $20: 14: 30.76,41: 41: 41.64$ & BIC610-0147 & $-0.9 \pm 0.25$ \\
\hline
\end{tabular}

Notes. The full table of 993 records is available at the CDS.

ellipse was contained in the $325 \mathrm{MHz}$ ellipse. For partial cases, we registered the percentage of overlapping area $(O A)$.

We then studied the $610 \mathrm{MHz}$ ellipse/s that was/were related to each single $325 \mathrm{MHz}$ ellipses and calculated a corresponding $610 \mathrm{MHz}$ contributing flux $S C_{2}$ that we used in the spectral index expression in the following way. For full cases, we considered $S C_{2}=S_{2}$. For partial cases, we set $S C_{2}=S_{2}$ when $O A \geq 70 \%, S C_{2}=0.5 S_{2}$ when $70 \%>O A>30 \%$, and
$S C_{2}=0$ elsewhere. We found that 993 sources at $325 \mathrm{MHz}$ overlap one or more $610 \mathrm{MHz}$ sources, and we computed the corresponding spectral indices by considering for each source at $325 \mathrm{MHz}$ all the overlapping sources at $610 \mathrm{MHz}$ with the weights as explained above. Table 5 lists the $325 \mathrm{MHz}$ source with its central coordinates, the $610 \mathrm{MHz}$ source(s) that partially or fully overlap the former, and the spectral index $\alpha$ as derived from $S_{1}$ and $S C_{2}$ (a few entries; the full table is available at the 

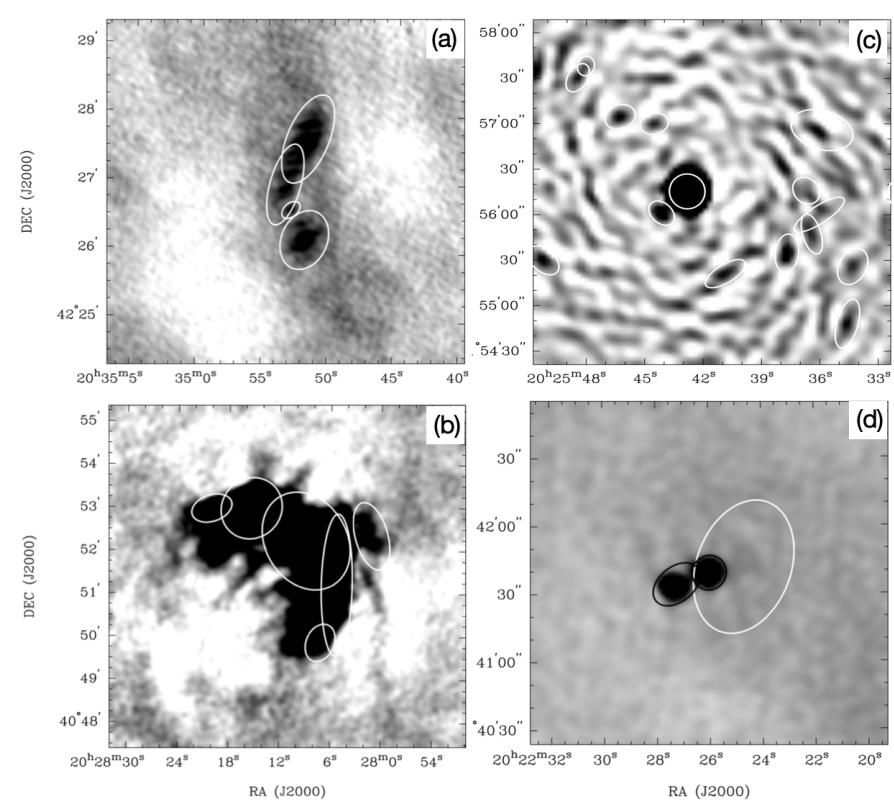

Fig. 5. Examples of discarded fits for the four cases given in Sect. 5, represented as white-line ellipses. (a): Filaments and/or diffuse emission at $610 \mathrm{MHz} .(b)$ : Strong or large sources that are ill represented by a combination of fits at $325 \mathrm{MHz}$. (c): Reduction artifacts at $325 \mathrm{MHz}$. $(d)$ : Fits of emission similar to the noise at $610 \mathrm{MHz}$; in this last case, the fits accepted as good fits are shown with black-line ellipses.

CDS). We present the spectral index uncertainty by error propagation in the very conservative case, that is, using the flux density errors given by PyBDSF combined with a $10 \%$ error for flux density scales (see Sect. 4).

To evaluate the probability of random matches when spectral indices are derived, we calculated the inverse of the number of sources per square degree over the area of the synthesized beam. At $325 \mathrm{MHz}$, we obtained that there will be one such a coincidence in 1700 sources. At $610 \mathrm{MHz}$, the probability of a random match is one source in 3230 . We found 1048 sources at $325 \mathrm{MHz}$, and 2796 sources at $610 \mathrm{MHz}$, therefore we assume that is very unlikely that unrelated sources overlap in our sample.

\section{Catalog properties}

\subsection{Detections, flux densities, and noise levels}

The catalog comprises 1048 sources at $325 \mathrm{MHz}$ and 2796 sources at $610 \mathrm{MHz}$ with flux densities greater than $7 \sigma$; here $\sigma$ represents the local $\mathrm{rms}$ noise at the source surroundings. The sources are characterized by their integrated and peak flux densities with corresponding errors, major and minor axes, and the position angle of a fitted ellipse, also with their errors.

Figure 6 displays the distribution of the flux density of the sources detected at the $325 \mathrm{MHz}$ band. In the lower panel we present a zoom on the flux interval $0-30 \mathrm{mJy}$, which contains $80 \%$ of the sources. The corresponding histograms for $610 \mathrm{MHz}$ appear in Fig. 7, with $91 \%$ of the sources with fluxes up to $30 \mathrm{mJy}$. At both bands, the effect of favoring higher resolution (meanwhile outlining more discrete sources) by means of the weighting scheme is appreciable as a majority of sources with lower fluxes. The detail for the sources with lower flux shows the typical decrease with flux (see, e.g., Fig. 3 in Zoonematkermani et al. 1990). The lower number of sources
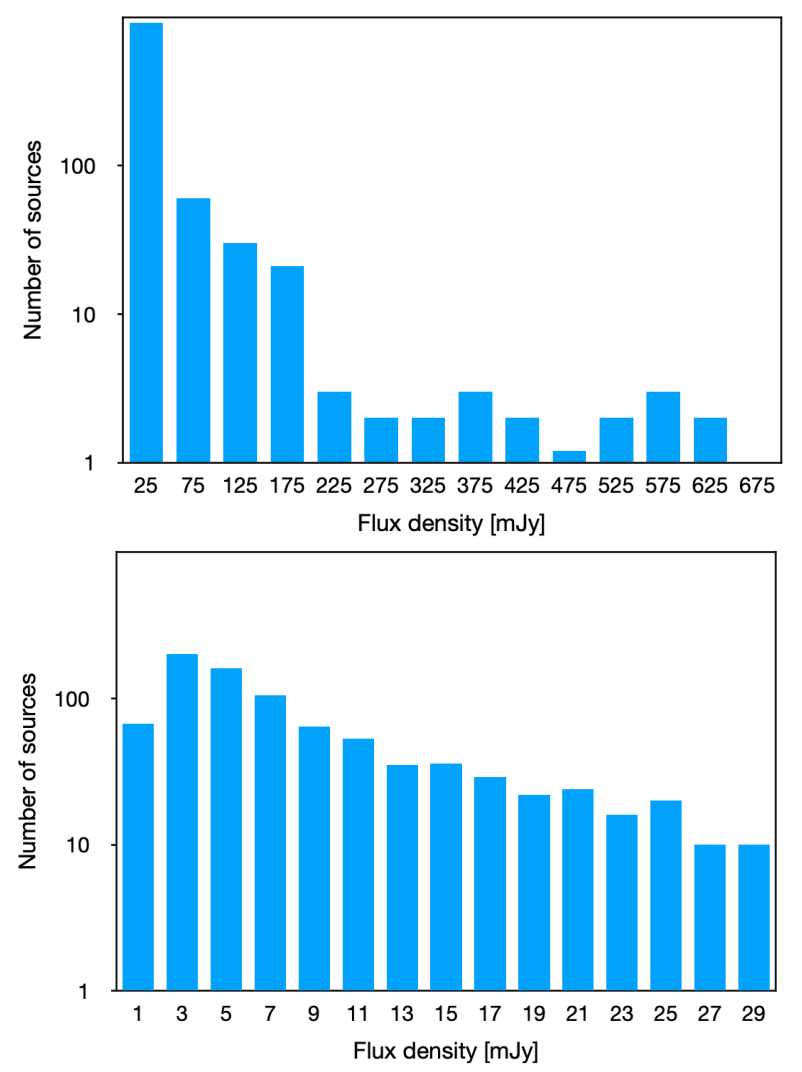

Fig. 6. Top: number of sources as a function of their integrated flux density for $99.71 \%$ of the sources cataloged at $325 \mathrm{MHz}$. Bottom: same for flux densities up to $30 \mathrm{mJy}$ (841 sources out of 1048, i.e., $80 \%$ ).

up to $5 \mathrm{mJy}$ at $325 \mathrm{MHz}$ is probably the effect of the detection threshold at this band ( $\simeq 2 \mathrm{mJy}$ on average).

We compared the number of sources we detected with the results from other studies. At the $325 \mathrm{MHz}$ band, the survey by Taylor et al. (1996), carried out with the Westerbork Synthesis Radio Telescope (WSRT) at $327 \mathrm{MHz}$, reported 3984 sources over a detection threshold of $10 \mathrm{mJy}$, an area of $160 \mathrm{sq}$ deg, and an angular resolution $\geq 1^{\prime}$, which means a ratio $R$ of 24.9 sources per square degree. At a similar frequency, we obtained 453 sources with fluxes above $10 \mathrm{mJy}$, thus a ratio of 40.1 sources per square degree. The difference can be explained by the larger beam that was used by the former survey, which is six times larger than the synthesised beam we used because some nearby GMRT sources will be seen as one WSRT source. A quick comparison with the detections reported by Setia Gunawan et al. (2003) (synthezised beam larger than five times that of this research) found that more than $90 \%$ of their sources that are present in the area are in common.

The VLA FIRST survey (Becker et al. 1995), performed at $20 \mathrm{~cm}$, found 946432 sources above a detection threshold of $1 \mathrm{mJy}(0.15 \mathrm{mJy} \mathrm{rms})$ using angular resolution images of $\sim 5^{\prime \prime}$ over an area of $10575 \mathrm{sq} \mathrm{deg}$, and then $R=89.5$. The FIRST detection limit corresponds to a value of $1.8 \mathrm{mJy}$ when it is scaled to the $610 \mathrm{MHz}$ band using a spectral index of -0.7 . In our catalog, almost 1800 sources showed flux densities higher than $1.8 \mathrm{mJy}$ and $R=91.4$, which agrees very well with the results from Becker et al. (1995).

For the rms per pixel at each band, we present in Fig. 8 the histograms showing the distribution of the rms in the fields we observed as obtained when the PyBDSF routines were applied at both bands. This rms value is estimated by PyBDSF near each 

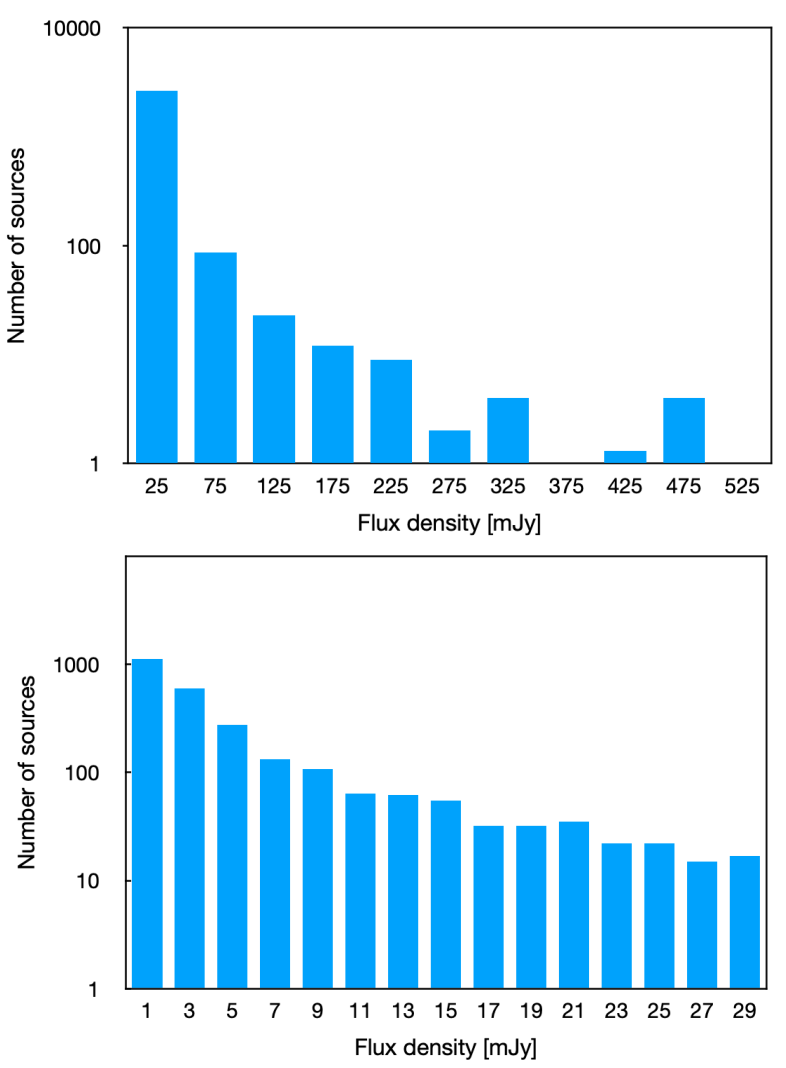

Fig. 7. Top: number of sources as a function of their integrated flux density for $99.75 \%$ of the sources cataloged at $610 \mathrm{MHz}$. Bottom: same for flux densities up to $30 \mathrm{mJy}$ ( 2565 sources out of 2796 , i.e., $91 \%$ ).

source before fitting. At $325 \mathrm{MHz}, 88 \%$ of the pixels show an rms of up to $0.5 \mathrm{mJy} \mathrm{beam}^{-1}$. At $610 \mathrm{MHz}, 80 \%$ of the pixels show an rms of up to $0.2 \mathrm{mJy}^{-1}$ beam $^{-1}$. We also compared these rms values and the rms values of a residual image from which the contribution of the fitted sources was subtracted. The results are presented in Fig. 9. The rms values decrease considerably, as expected.

\subsection{Resolved and unresolved sources}

To distinguish between resolved and unresolved sources, we plot in Fig. 10 the ratio of the total (integrated) flux over the peak flux for circular sources $\left(\theta_{1} / \theta_{2}<1.05,418\right.$ sources) at $610 \mathrm{MHz}$. The ratio remains below 1.25 out to $6.6^{\prime \prime}$, which we adopt as the dividing line between resolved and unresolved sources. This value by coincidence is the size of the synthesised beam plus a $10 \%$ error at this frequency. We applied the same criterion based on the ratio value of 1.25 for the sources detected at $325 \mathrm{MHz}$. The distribution of the mean axes (sizes) of the cataloged sources, in the form of the average of the major and minor axes of each ellipse, is shown in Figs. 11 and 12.

\subsection{Source multiplicity}

During the visual inspection process of all sources we found at both bands, we marked those that are characterized by adjacent emission components, fit by distinct Gaussian functions. In many of them, even a bridge linking components was clearly seen. Following the technique by Magliocchetti et al. (1998) and Huynh et al. (2005), we listed the sources that presented a companion up to $2^{\prime}$, and distinguished the pairs (source+companion) where the flux density ratio (brighter over weaker) remained
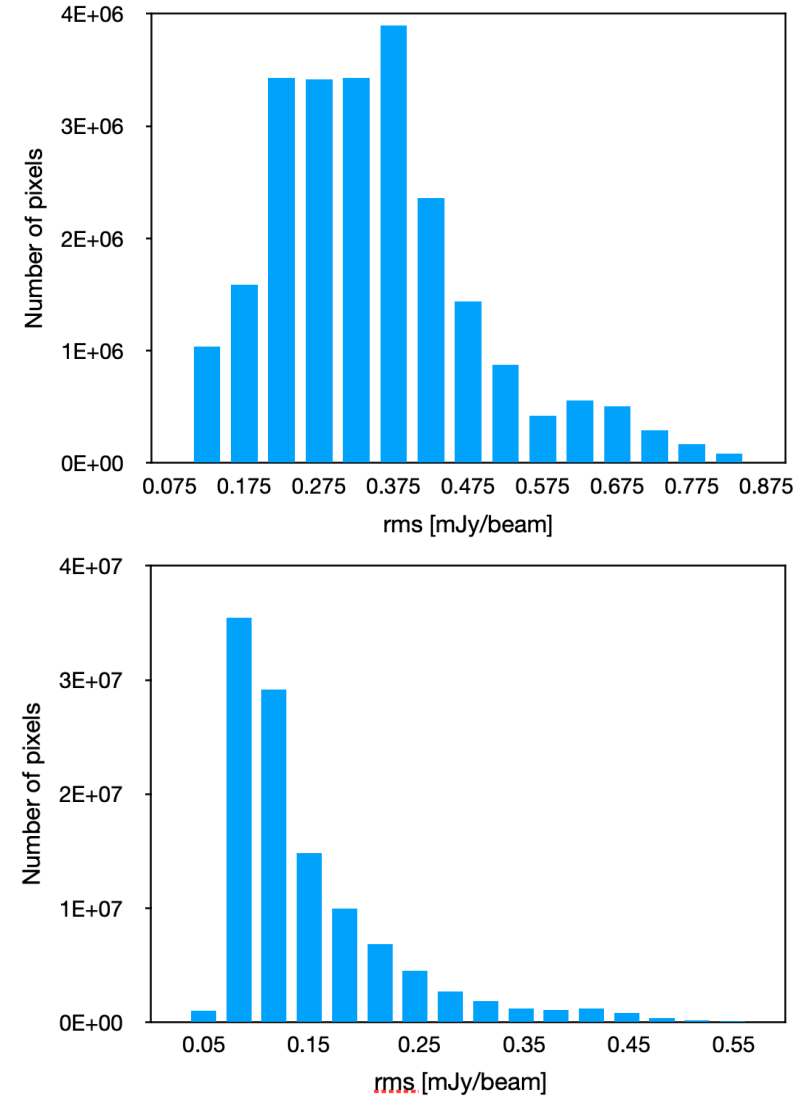

Fig. 8. Distribution of the rms in the mosaics at $325 \mathrm{MHz}$ (top panel) and at $610 \mathrm{MHz}$ (bottom panel).
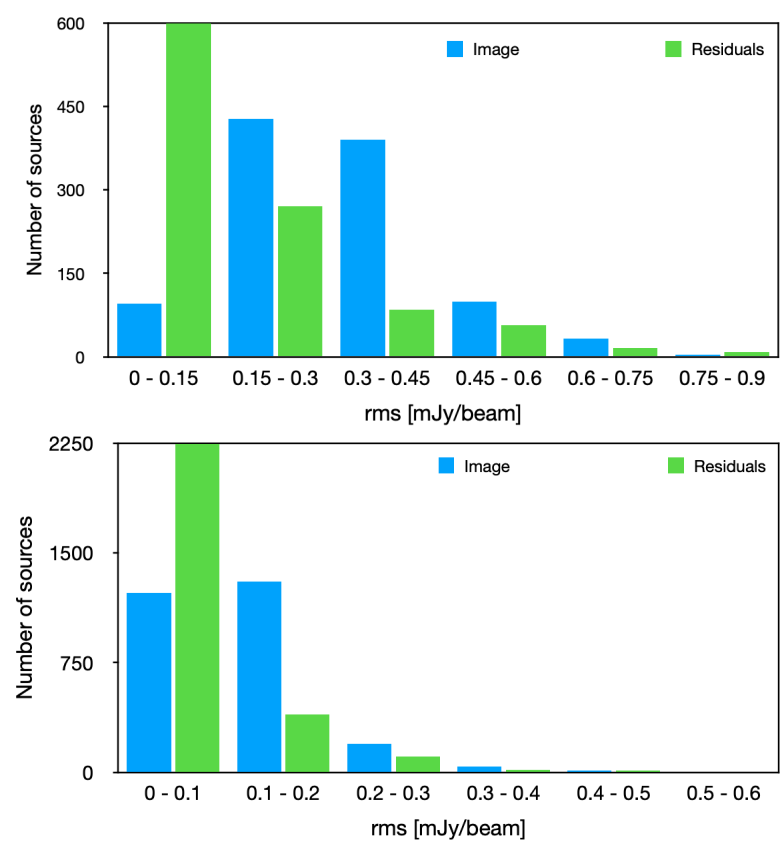

Fig. 9. Distribution of the rms in the mosaics before (light blue bars) and after source extraction (green bars) at $325 \mathrm{MHz}$ (top panel) and at $610 \mathrm{MHz}$ (bottom panel).

below 4. Figure 13 represents these groups in the plane of the sum of the fluxes $(F S)$ of the components for each pair versus the separation $(x)$ between components. 

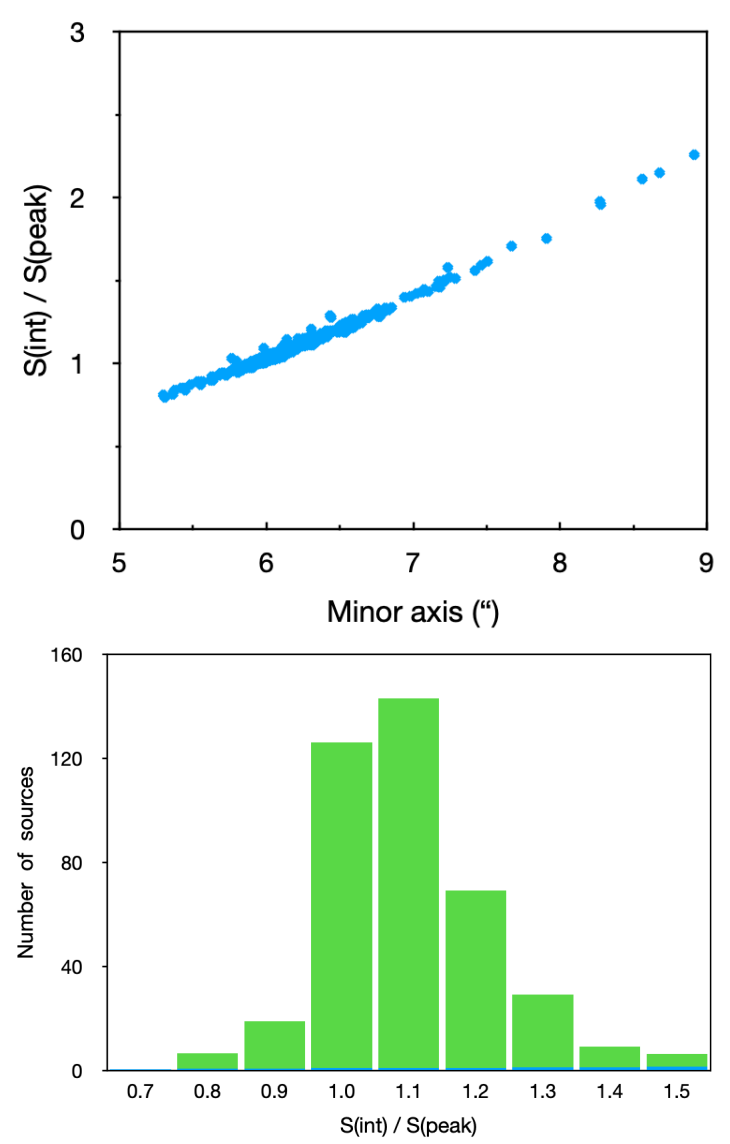

Fig. 10. Top panel: ratio of total flux over peak flux as a function of their minor axis for sources with $\theta_{1} / \theta_{2} \leq 1.05$, cataloged at $610 \mathrm{MHz}$. A power fit yields $S_{\text {int }} / S_{\text {peak }}=1.25$ at $\theta_{2}=6.6^{\prime \prime}$. Bottom panel: distribution of the flux ratio of the same group of sources.

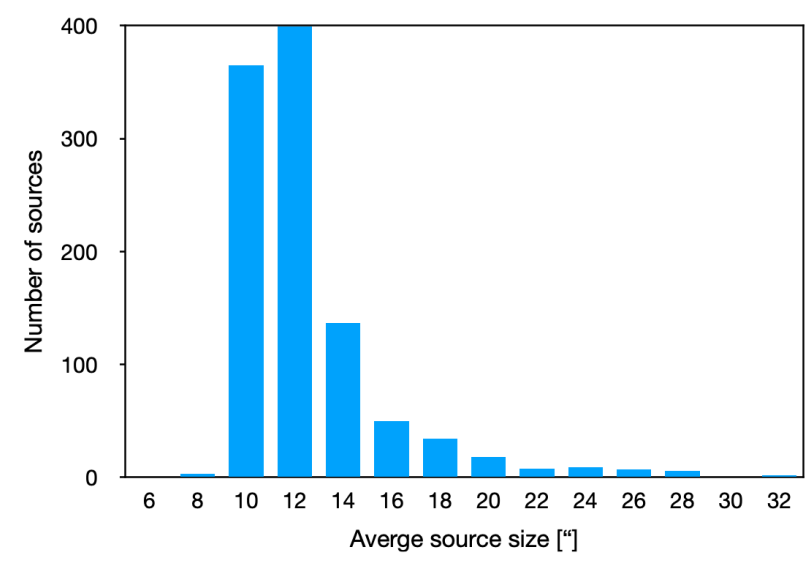

Fig. 11. Distribution of the average axis $[0.5 \times$ (major axis + minor axis)] of the ellipses representing the sources at $325 \mathrm{MHz}$.

Two areas are clearly visible in the plots at both bands, depending on whether they contain visually checked double pairs. Previous works have found that the limit between the areas can be described by $F S \propto x^{2}$. The data presented here appear to be better confined with an exponential of 3.5; see Fig. 13, where we plot the two limiting lines. In principle, we can infer that the components of the pairs in the left areas are more likely to be physically related.

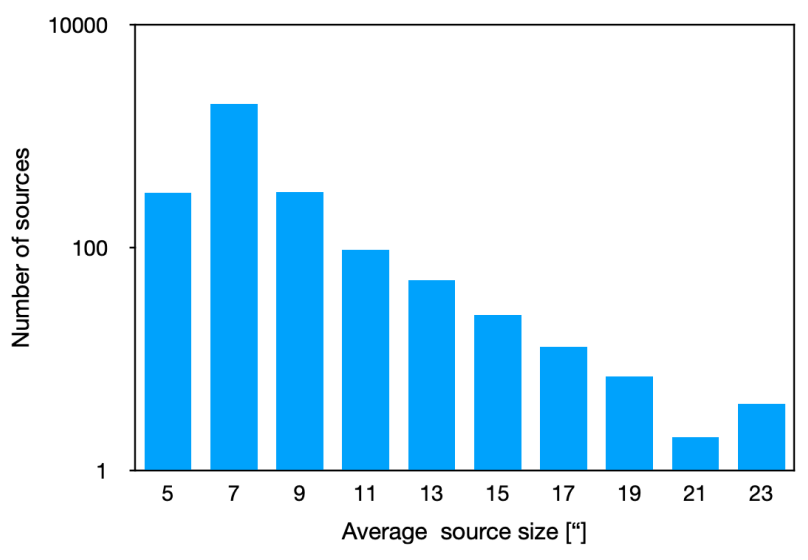

Fig. 12. Distribution of the average axis $[0.5 \times$ (major axis + minor axis)] of the ellipses representing the sources at $610 \mathrm{MHz}$.
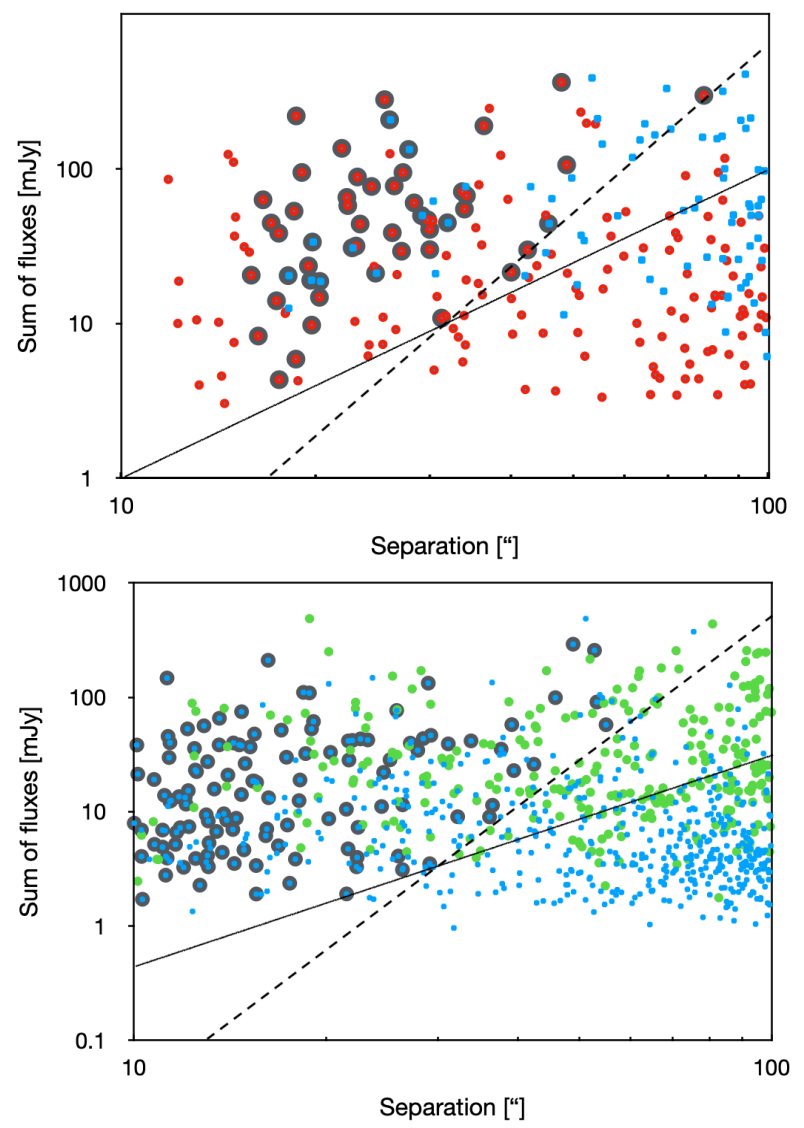

Fig. 13. Sum of the flux densities from pairs of nearby sources $(F S)$ vs. source separation $(x)$. Top panel: $325 \mathrm{MHz}$ sources; in red, pairs of sources whose flux ratio (integrated/peak) is below 4; in blue, the rest; larger dark gray circles: pairs of confirmed double sources (see text). Dashed line: $(x / 16)^{3.5}$. Thin solid line: $(x / 10)^{2}$. Bottom panel: for $610 \mathrm{MHz}$ sources; in green, pairs of sources whose flux ratio is below 4 ; in blue, the rest; larger dark gray circles: pairs of double sources (see text). Dashed line: $(x / 20)^{3.5}$. Thin solid line: $(x / 16)^{2}$.

\subsection{Considerations of the spectral indices}

In the case of the spectral index distribution of the about one thousand sources that are detected at two bands in our catalog, the pronounced maximum at $\alpha=-1$ confirms the nonthermal nature of most of the sources, see Figs. 14 and 15. The median error in $\alpha$ is 0.29 . The spectral index values span from -3.06 


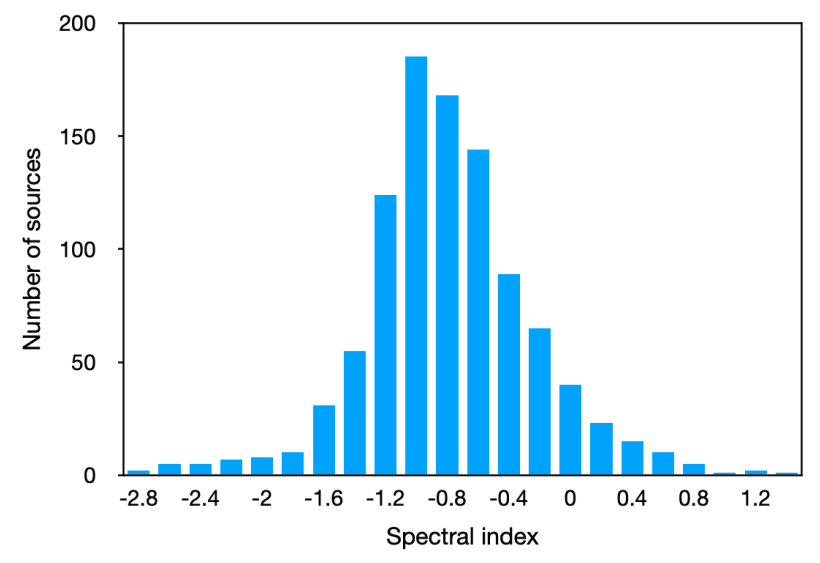

Fig. 14. Distribution of spectral indices corresponding to the sources detected at both frequency bands (see Table 5).

to +1.41 . Only 4 out of 993 values are below -2.5 . Variability can be one of the reasons for the extreme absolute values of the index: the two frequency data were taken at different times. They might also arise because we systematically considered the fluxes for sources that do not fully overlap.

The uncertainties on the spectral indices are somewhat large, which is due to the conservative error on the flux density and because the two frequencies lie only about a factor of two apart. However, this should be sufficient to broadly categorize the sources as thermal, nonthermal, or pick up sources with very steep spectra.

The 993 sources with a spectral index value detected at the $325 \mathrm{MHz}$ band correspond to 1065 sources at the $610 \mathrm{MHz}$ band: at this latter frequency, the synthesized beam is smaller, and we found two or even three $610 \mathrm{MHz}$ sources that overlap the same $325 \mathrm{MHz}$ object; see Table 5. The surveyed area at $325 \mathrm{MHz}$ totaled 23486672 pixels with signal (pixel size = $\left.2.5^{\prime \prime} \times 2.5^{\prime \prime}\right)$, or $11.3 \mathrm{sq} \mathrm{deg}$. At $610 \mathrm{MHz}, 113370869$ pixels with signal accounted for $19.7 \mathrm{sq} \mathrm{deg}$ (pixel size $\left.=1.5^{\prime \prime} \times 1.5^{\prime \prime}\right)$. In the same area covered by the $325 \mathrm{MHz}$ mosaic, 1796 sources at $610 \mathrm{MHz}$ (out of the total number of 2796) were fit, thus the ratio of source-fits at 610 to $325 \mathrm{MHz}$ is 1.71 . This can be explained by sensitivity limitations, considering that at $610 \mathrm{MHz}$ we have better angular resolution and lower noise, which allows us to detect thermal sources that will remain undetected at $325 \mathrm{MHz}$ also because they are fainter, except for the cases when we picked up more than one source counterpart of a single $325 \mathrm{MHz}$ source at $610 \mathrm{MHz}$.

\section{Search for counterparts}

After the catalog of 325 and $610 \mathrm{MHz}$ sources was completed, we searched for nearby objects as possible counterparts to the $(1048+2796=) 3844$ entries. We used the Simbad database ${ }^{5}$, with two input tables: one containing the coordinates of the records of the $325 \mathrm{MHz}$ sources, and a second table with those of the $610 \mathrm{MHz}$ sources. The search radius was set as the semimajor axis of the ellipse fit of each record. We found possible counterparts for 85 sources at $325 \mathrm{MHz}$ and for 138 sources at $610 \mathrm{MHz}$, where more than one nearby object was found for some of the sources. We studied all possible counterparts in each case and also verified that the nearby objects were inside the fitted ellipse, that is, taking the semiminor axis and position angle

\footnotetext{
5 http://simbad.u-strasbg.fr/simbad/sim-fid
}

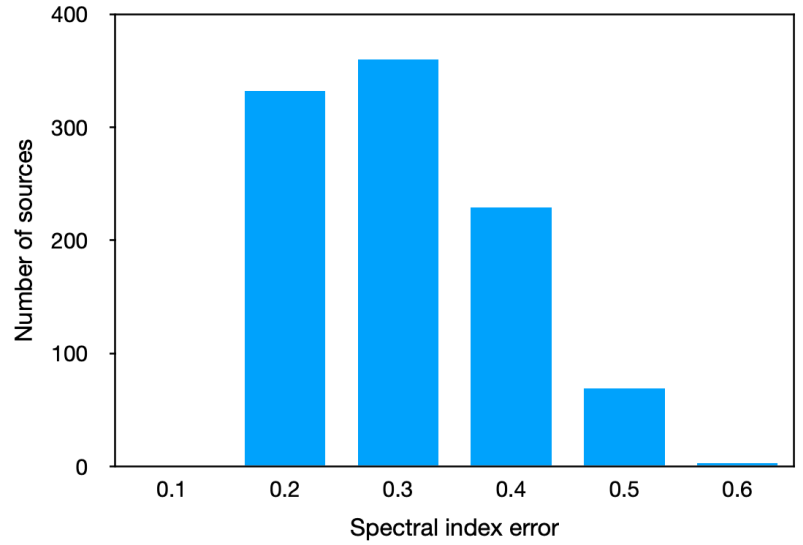

Fig. 15. Distribution of spectral index errors corresponding to the sources detected at both frequency bands (see Table 5).

of the source fit into consideration. We gathered the findings in Table A.1, which presents possible counterparts for 5 sources that are only detected at $325 \mathrm{MHz}$, for 52 sources that are only detected at $610 \mathrm{MHz}$, and for 86 sources that are detected at both bands, ordered by right ascension. The angular distance $d$ between the GMRT source and the potential counterpart and the spectral index, if applicable, is also listed. By searching the literature, we investigated the nature of the potential counterpart, and propose the more plausible object that could be associated with the GMRT sources reported here whenever possible, along with its reference or, in the worst case, the reference of flux measured at other wavelength(s). In the cases with preexisting $325 \mathrm{MHz}$ observations, we quote no counterpart because our observations superseded them in sensitivity or also in angular resolution. In addition to information from the surveys mentioned in Sect. 2, valuable material was found in Vollmer et al. (2010), who compiled flux values of sources at the radio range including those of the Cygnus region relevant here. An exception is made for fluxes at the $610 \mathrm{MHz}$ band, for which no previous data were found. In this sense, our catalog completes many radio spectra and provides $610 \mathrm{MHz}$ flux values of $\sim 2800$ sources for the first time.

Of the sources with possible counterparts that were detected at both frequencies, $16 \%$ have flat or positive spectral index (nominally, $\alpha>-0.2$ ). Their counterparts are mostly either stars, young stellar objects, or radio sources detected at higher frequencies.

At the $325 \mathrm{MHz}$ band, $8 \%$ of all the $325 \mathrm{MHz}$ cataloged sources have a possible counterpart. At $610 \mathrm{MHz}, 5 \%$ of all the $610 \mathrm{MHz}$ cataloged sources have a possible counterpart. These low percentages can be explained considering that at the observed bands, decimeter wavelengths, we mostly sample nonthermal sources, at which high-energy (HE) processes presumably take place. These might be potential counterparts to the HE sources; but the angular resolutions and sensitivities for instruments working at HE ranges are higher and lower, respectively, precluding successful cross-identifications between radio and HE sources.

Preliminary results of a study focused on the unresolved unidentified sources with negative spectral index ( $~ 340$ sources of the present catalog), such as was performed by Chakraborty et al. (2020), indicated that differential source counts in the distribution trend of extragalactic sources result from other surveys or catalogs. However, we recall that the observed fields are in the surroundings of the Galactic plane, dense in Galactic sources, both of thermal and nonthermal nature. A detailed investigation to 
distinguish in which proportion, and more interesting, from which types of objects, Galactic and extragalactic sources contribute etc. is beyond the scope of this paper.

\section{Related studies and prospects}

The GMRT observations that gave rise to our catalog allowed us simultaneously to carry out research on individual populations of astronomical objects. Specifically, different types of objects that can produce nonthermal emission were or are being studied in separated investigations: AGNs and two-lobed sources, counterparts to HE sources, massive early-type stars (Benaglia et al. 2020), protoplanetary disk-like sources (Isequilla et al. 2019) and young stellar objects (Isequilla et al. 2020). Finally, the survey images will be presented elsewhere. Future work includes the scrutiny of sources between 3 and $7 \sigma$. The corresponding source extraction, after a thorough validation process, of such a large area with the angular resolution of a few arcseconds provided by the GMRT data at decimeter frequencies will certainly reveal a plethora of interesting objects and powerful statistical results of the nonthermal sky.

Acknowledgements. The authors are grateful to the referee, whose comments and suggestions resulted in improving the analysis and presentation of the article. The GMRT is operated by the National Centre for Radio Astrophysics of the Tata Institute of Fundamental Research. We thank the staff of the GMRT that made these observations possible. PB acknowledges support from ANPCyT PICT 0773-2017, and the contacts at NCRA, Pune for a very pleasant stay. ICCH acknowledges the support of the Department of Atomic Energy, Government of India, under the project 12-R\&D-TFR-5.02-0700. This research has made use of the SIMBAD database, operated at CDS, Strasbourg, France, and of NASA's Astrophysics Data System bibliographic services.

\section{References}

Abeysekara, A. U., Archer, A., Aune, T., et al. 2018, ApJ, 861, 134

Albacete Colombo, J. F., Flaccomio, E., Micela, G., Sciortino, S., \& Damiani, F. 2007, A\&A, 464, 211

Anderson, L. D., Armentrout, W. P., Johnstone, B. M., et al. 2015, ApJS, 221, 26

Araudo, A. T., Romero, G. E., Bosch-Ramon, V., \& Paredes, J. M. 2007, A\&A, 476,1289

Becker, R. H., White, R. L., \& Helfand, D. J. 1995, ApJ, 450, 559

Benaglia, P., \& Romero, G. E. 2003, A\&A, 399, 1121

Benaglia, P., Romero, G. E., Martí, J., Peri, C. S., \& Araudo, A. T. 2010, A\&A, 517, L10

Benaglia, P., del Palacio, S., Ishwara-Chandra, C. H., et al. 2019, A\&A, 625, A99

Benaglia, P., De Becker, M., Ishwara-Chandra, C. H., Intema, H., \& Isequilla, N. 2020, PASA, 38, 1

Bosch-Ramon, V., Romero, G. E., Araudo, A. T., \& Paredes, J. M. 2010, A\&A, 511, A8

Brinkmann, W., Siebert, J., Feigelson, E. D., et al. 1997, A\&A, 323, 739

Bulgarelli, A., Fioretti, V., Parmiggiani, N., et al. 2019, A\&A, 627, A13

Chakraborty, A., Roy, N., Wang, Y., et al. 2020, MNRAS, 492, 2236

Chandra, P., Ray, A., \& Bhatnagar, S. 2004, ApJ, 612, 974

Chen, A. M., Takata, J., Yi, S. X., Yu, Y. W., \& Cheng, K. S. 2019, A\&A, 627 A87

Clegg, A. W., Cordes, J. M., Simonetti, J. M., \& Kulkarni, S. R. 1992, ApJ, 386, 143

Colley, D. 1980, MNRAS, 192, 377

Comerón, F., Pasquali, A., Rodighiero, G., et al. 2002, A\&A, 389, 874

Condon, J. J., Cotton, W. D., Greisen, E. W., et al. 1998, AJ, 115, 1693

Cutri, R. M., Skrutskie, M. F., van Dyk, S., et al. 2003, VizieR Online Data Catalog, II/246

De Becker, M., \& Raucq, F. 2013, A\&A, 558, A28

del Palacio, S., Bosch-Ramon, V., Müller, A. L., \& Romero, G. E. 2018, A\&A, 617, A13

del Valle, M. V., \& Pohl, M. 2018, ApJ, 864, 19

Douglas, J. N., Bash, F. N., Bozyan, F. A., Torrence, G. W., \& Wolfe, C. 1996 AJ, 111, 1945

Elyajouri, M., Monreal-Ibero, A., Remy, Q., \& Lallement, R. 2016, ApJS, 225, 19
Garwood, R. W., Perley, R. A., Dickey, J. M., \& Murray, M. A. 1988, AJ, 96, 1655

Greaves, J. 2004, MNRAS, 355, 585

Hartman, R. C., Bertsch, D. L., Bloom, S. D., et al. 1999, ApJS, 123, 79

Haslam, C. G. T., Salter, C. J., Stoffel, H., \& Wilson, W. E. 1982, A\&AS, 47, 1

Hermsen, W., Swanenburg, B. N., Bignami, G. F., et al. 1977, Nature, 269, 494

Higgs, L. A., Wendker, H. J., \& Landecker, T. L. 1994, A\&A, 291, 295

Høg, E., Fabricius, C., Makarov, V. V., et al. 2000, A\&A, 355, L27

Huynh, M. T., Jackson, C. A., Norris, R. P., \& Prandoni, I. 2005, AJ, 130, 1373 Immer, K., Brunthaler, A., Reid, M. J., et al. 2011, ApJS, 194, 25

Intema, H. T. 2014, SPAM: Source Peeling and Atmospheric Modeling (Astrophysics Source Code Library)

Isequilla, N. L., Fernández-López, M., Benaglia, P., Ishwara-Chandra, C. H., \& del Palacio, S. 2019, A\&A, 627, A58

Isequilla, N. L., Benaglia, P., Ishwara-Chandra, C. H., \& Intema, H. 2020, Bol. Asoc. Argent. Astron., 61B, 1

Ishwara-Chandra, C. H., Benaglia, P., De Becker, M., \& Tej, A. 2019, Bull. Soc. Roy. Sci. Liege, 88, 166

Johnston, K. G., Shepherd, D. S., Robitaille, T. P., \& Wood, K. 2013, A\&A, 551, A43

Knödlseder, J. 2000, A\&A, 360, 539

Kohoutek, L. 2001, A\&A, 378, 843

Kryukova, E., Megeath, S. T., Hora, J. L., et al. 2014, AJ, 148, 11

Lu, X., Zhang, Q., Liu, H. B., Wang, J., \& Gu, Q. 2014, ApJ, 790, 84

Magliocchetti, M., Maddox, S. J., Lahav, O., \& Wall, J. V. 1998, MNRAS, 300, 257

Mahy, L., Rauw, G., De Becker, M., Eenens, P., \& Flores, C. A. 2013, A\&A, 550, A27

Martí, J., Pérez-Ramírez, D., Luque-Escamilla, P., et al. 2006, A\&A, 451, 1037

Melikian, N. D., Tamazian, V. S., Karapetian, A. A., Samsonian, A. L., \& Kostandian, G. R. 2011, Astrophysics, 54, 203

Massaro, F., D’Abrusco, R., Giroletti, M., et al. 2013, ApJS, 207, 4

Mitronova, S. N., Karachentsev, I. D., Karachentseva, V. E., Jarrett, T. H., \& Kudrya, Y. N. 2004, Bull. Spec. Astrophys. Obs., 57, 5

Montes, V. A., Hofner, P., Anderson, C., \& Rosero, V. 2015, ApJS, 219, 41

Neria, C., Gómez, Y., \& Rodríguez, L. F. 2010, Rev. Mex. Astron. Astrofis., 46, 253

Panessa, F., Tarchi, A., Castangia, P., et al. 2015, MNRAS, 447, 1289

Paredes, J. M., Martí, J., Ishwara-Chandra, C. H., et al. 2008, A\&A, 482, 247

Parthasarathy, M., Jain, S. K., \& Bhatt, H. C. 1992, A\&A, 266, 202

Paunzen, E. 2015, A\&A, 580, A23

Price-Whelan, A. M., Hogg, D. W., Rix, H.-W., et al. 2018, AJ, 156, 18

Purcell, C. R., Hoare, M. G., Cotton, W. D., et al. 2013, ApJS, 205, 1

Ramachandran, V., Das, S. R., Tej, A., et al. 2017, MNRAS, 465, 4753

Rauw, G. 2011, A\&A, 536, A31

Reddish, V. C., Lawrence, L. C., \& Pratt, N. M. 1966, Publ. Roy. Obs. Edinburgh, 5,111

Reipurth, B., \& Schneider, N. 2008, Star Formation and Young Clusters in Cygnus, ed. B. Reipurth (ASP), 36

Rengelink, R. B., Tang, Y., de Bruyn, A. G., et al. 1997, A\&AS, 124, 259

Rodríguez-Kamenetzky, A., Carrasco-González, C., González-Martín, O., et al. 2019, MNRAS, 482, 4687

Romero, G. 2008, in Gamma Rays from Star Forming Regions, ed. F. A. Aharonian (AIP), 97

Romero, G. E., Torres, D. F., Kaufman Bernadó, M. M., \& Mirabel, I. F. 2003, A\&A, 410, L1

Sánchez-Monge, Á., Palau, A., Estalella, R., Beltrán, M. T., \& Girart, J. M. 2008, A\&A, 485, 497

Scaife, A. M. M., \& Heald, G. H. 2012, MNRAS, 423, L30

Setia Gunawan, D. Y. A., de Bruyn, A. G., van der Hucht, K. A., \& Williams, P. M. 2003, ApJS, 149, 123

Solin, O., Ukkonen, E., \& Haikala, L. 2012, A\&A, 542, A3

Swarup, G., Ananthakrishnan, S., Kapahi, V. K., et al. 1991, Curr. Sci., 60, 95

Taylor, A. R., Goss, W. M., Coleman, P. H., van Leeuwen, J., \& Wallace, B. J. 1996, ApJS, 107, 239

The Fermi-LAT collaboration 2020, ApJS, 247, 33

Urquhart, J. S., Hoare, M. G., Purcell, C. R., et al. 2009, A\&A, 501, 539

Uyanıker, B., Fürst, E., Reich, W., Aschenbach, B., \& Wielebinski, R. 2001, A\&A, 371, 675

Verbeek, K., Groot, P. J., Scaringi, S., et al. 2012, MNRAS, 426, 1235

Véron-Cetty, M. P., \& Véron, P. 2010, A\&A, 518, A10

Vollmer, B., Gassmann, B., Derrière, S., et al. 2010, A\&A, 511, A53

Wendker, H. J., Higgs, L. A., \& Landecker, T. L. 1991, A\&A, 241, 551

Williams, P. K. G., Bower, G. C., Croft, S., et al. 2013, ApJ, 762, 85

Wright, N. J., Drake, J. J., Drew, J. E., et al. 2012, ApJ, 746, L21

Zhang, Q., Claus, B., Watson, L., \& Moran, J. 2017, ApJ, 837, 53

Zoonematkermani, S., Helfand, D. J., Becker, R. H., White, R. L., \& Perley, R. A. 1990, ApJS, 74, 181 
P. Benaglia et al.: Cygnus survey with the Giant Metrewave Radio Telescope at 325 and $610 \mathrm{MHz}$ : the catalog

\section{Appendix A: Additional table}

Table A.1. Counterparts of detected sources at $325 \mathrm{MHz}$ and $610 \mathrm{MHz}$.

\begin{tabular}{|c|c|c|c|c|c|c|c|c|}
\hline $\begin{array}{l}325-I D \\
\text { BIC325- }\end{array}$ & $\begin{array}{r}\text { 610-ID } \\
\text { BIC610- }\end{array}$ & $\begin{array}{l}\text { Spectral } \\
\text { index }\end{array}$ & $\begin{array}{c}\mathrm{RA}_{\mathrm{J} 2000} \\
(\mathrm{~h}, \mathrm{~m}, \mathrm{~s})\end{array}$ & $\begin{array}{l}\operatorname{Dec}_{J 2000} \\
\left(\operatorname{deg},,^{\prime},{ }^{\prime}\right)\end{array}$ & $\begin{array}{c}d \\
\left({ }^{\prime \prime}\right)\end{array}$ & $\begin{array}{c}\text { SIMBAD nearest } \\
\text { source }\end{array}$ & Reference & $\begin{array}{l}\text { Information on } \\
\text { possible nature }\end{array}$ \\
\hline- & 0045 & - & $20: 12: 57.27$ & $41: 51: 49.34$ & 4.6 & 2MFGC 15386 & Mitronova et al. (2004) & Disk-like galaxy \\
\hline - & 0093 & - & $20: 13: 47.77$ & $41: 10: 02.13$ & 7.6 & CXO J201348.3+411007.1 & Montes et al. (2015) & X-ray source \\
\hline 0006 & 0124 & $-1.0 \pm 0.1$ & $20: 14: 18.68$ & $41: 18: 11.26$ & 5.4 & CXO J201419.1+411813.4 & Montes et al. (2015) & Star-forming region \\
\hline- & 0217 & - & $20: 15: 21.60$ & $40: 34: 43.93$ & 6.8 & PN KjPn 2 & Kohoutek (2001) & Planetary nebula \\
\hline 0107 & 0429 & $-1.0 \pm 0.3$ & $20: 17: 54.12$ & $41: 24: 14.49$ & 5.3 & J201754.59+412413.98 & Kryukova et al. (2014) & IR source \\
\hline 0119 & 0462 & $-1.6 \pm 0.2$ & 20:18:07.79 & $41: 10: 41.13$ & 5.2 & FGL J2018.1+4111 & Abeysekara et al. (2018) & $\gamma$-ray source \\
\hline 0134 & 0531 & $-2.4 \pm 0.1$ & $20: 18: 38.18$ & $40: 41: 00.42$ & 3.7 & MSX6C G078.0875+02.6408 & Panessa et al. (2015) & Seyfert galaxy \\
\hline- & 0543 & - & $20: 18: 42.69$ & $44: 17: 34.13$ & 2.2 & NVSS J201842+441736 & Vollmer et al. (2010) & \\
\hline- & 0551 & - & $20: 18: 46.65$ & $42: 20: 04.91$ & 8.2 & NVSS J201847+422010 & Condon et al. (1998) & \\
\hline- & 0582 & - & 20:19:02.55 & $40: 18: 26.02$ & 4.1 & WSRTGP 2017+4009 & Vollmer et al. (2010) & \\
\hline 0151 & 0624 & $-0.2 \pm 0.3$ & $20: 19: 19.30$ & $40: 54: 51.52$ & 7.8 & MTK2011-F1 14 & Melikian et al. (2011) & Emission-line star \\
\hline 0162/0163/0164 & $0665 / 0666 / 0672$ & $-1.0 \pm 0.2$ & $20: 19: 36.50$ & $40: 58: 50.00$ & $\sim 10$ & NGR2010-VLAN 2,3 & Neria et al. (2010) & UC HII region \\
\hline 0165 & 0676 & $+0.9 \pm 0.1$ & $20: 19: 38.89$ & $40: 56: 36.21$ & 1.3 & IRAS $20178+4046-$ VLAN 4 & Neria et al. (2010) & UC HII region \\
\hline 0170 & 0690 & $-1.0 \pm 0.1$ & $20: 19: 49.33$ & $42: 00: 12.26$ & 5.0 & J201949.77+420011.20 & Kryukova et al. (2014) & YSO \\
\hline- & 0731 & - & 20:20:09.10 & $43: 40: 22.78$ & 2.0 & RX J2020.0+4357 & Brinkmann et al. (1997) & $\mathrm{X}$-ray source \\
\hline 0182 & 0757 & $+0.1 \pm 0.2$ & $20: 20: 18.62$ & $40: 58: 03.26$ & 5.3 & J202019.08+405802.18 & Kryukova et al. (2014) & YSO \\
\hline- & 0787 & - & $20: 20: 27.95$ & $43: 51: 13.88$ & 2.4 & WR 140 & De Becker \& Raucq (2013) & WR system \\
\hline 0196 & 0808 & $+0.3 \pm 0.1$ & $20: 20: 35.65$ & $40: 57: 54.84$ & 6.2 & J202036.15+405753.58 & Kryukova et al. (2014) & YSO \\
\hline$" \prime$ & " & " & $20: 20: 36.18$ & $40: 57: 53.08$ & 6.6 & NVSS J202036+405754 & Vollmer et al. (2010) & \\
\hline- & 0825 & - & $20: 20: 42.59$ & $42: 16: 46.54$ & 8.0 & MITG J2020+4216 & Vollmer et al. (2010) & \\
\hline 0200 & 0842 & $-0.0 \pm 0.3$ & $20: 20: 51.07$ & $41: 22: 05.96$ & 5.6 & J202051.55+412204.76 & Kryukova et al. (2014) & YSO \\
\hline- & 0861 & - & $20: 20: 57.67$ & $44: 41: 29.82$ & 0.9 & NVSS J202057+444130 & Vollmer et al. (2010) & \\
\hline- & 0894 & - & $20: 21: 18.24$ & $41: 19: 59.65$ & 5.0 & J202118.68+411958.86 & Kryukova et al. (2014) & YSO \\
\hline- & 0967 & - & $20: 21: 49.02$ & $44: 00: 37.37$ & 2.7 & 2MASX J20214907+4400399 & Véron-Cetty \& Véron (2010) & Seyfert galaxy \\
\hline- & 1049 & - & $20: 22: 17.22$ & $42: 24: 49.47$ & 10.4 & $18 \mathrm{P} 22$ & Vollmer et al. (2010) & \\
\hline 0263 & 1051 & $-0.1 \pm 0.2$ & $20: 22: 17.85$ & 43:53:01.09 & 8.3 & 2MASS J20221736+4353074 & Price-Whelan et al. (2018) & Red giant star \\
\hline- & 1112 & - & $20: 22: 44.55$ & $41: 45: 17.41$ & 5.9 & J202245.07+414517.98 & Kryukova et al. (2014) & YSO \\
\hline - & 1117 & - & $20: 22: 46.33$ & 41:07:00.44 & 5.8 & J202245.07+414517.98 & Kryukova et al. (2014) & YSO \\
\hline - & 1126 & - & $20: 22: 52.35$ & $44: 48: 20.56$ & 3.3 & BD +443444 & Elyajouri et al. (2016) & B8 star \\
\hline- & 1205 & - & $20: 23: 19.06$ & $43: 12: 44.08$ & 8.2 & IRAS $20216+4303$ & Vollmer et al. (2010) & \\
\hline 0343 & 1248 & $+0.1 \pm 0.1$ & $20: 23: 35.68$ & $41: 25: 26.43$ & 3.3 & $\mathrm{~J} 202335.81+412523.53$ & Kryukova et al. (2014) & YSO \\
\hline 0347 & 1253 & $-0.8 \pm 0.1$ & $20: 23: 39.05$ & 44:01:04.49 & 10.6 & $18 \mathrm{P} 25$ & Vollmer et al. (2010) & \\
\hline 0393 & 1329 & $-1.2 \pm 0.2$ & $20: 24: 11.53$ & $41: 43: 24.47$ & 9.1 & TYC 3160-519-1 & Høg et al. (2000) & Star \\
\hline 0390/0395 & 1334 & $-0.5 \pm 0.1$ & $20: 24: 15.43$ & $43: 22: 32.22$ & 5 & NVSS J202415+432235 & Vollmer et al. (2010) & \\
\hline 0434 & 1404 & $-0.6 \pm 0.3$ & $20: 24: 46.19$ & $42: 23: 13.16$ & 9.3 & BD+41 3737 & Paunzen (2015) & Star \\
\hline- & 1420 & - & $20: 24: 52.00$ & $40: 40: 25.21$ & 5.6 & G078.779+01.693 & Anderson et al. (2015) & HII region \\
\hline 0460 & 1447 & $-0.7 \pm 0.1$ & $20: 25: 00.59$ & $41: 48: 25.43$ & 6.1 & NVSS J202501+414829 & Condon et al. (1998) & \\
\hline 0490 & 1493 & $+0.6 \pm 0.1$ & $20: 25: 19.02$ & $43: 35: 19.40$ & 4.7 & J2025+4335 & Immer et al. (2011) & \\
\hline 0496 & 1503 & $-0.8 \pm 0.1$ & $20: 25: 22.74$ & $44: 19: 33.44$ & 1.4 & NVSS J202522+441934 & Condon et al. (1998) & \\
\hline 0503 & 1511 & $-0.8 \pm 0.2$ & $20: 25: 24.78$ & 41:03:19.48 & 3.6 & G079.151+01.830 & Solin et al. (2012) & Star-forming region \\
\hline 0526 & $1557 / 1563$ & $-1.1 \pm 0.1$ & $20: 25: 40.53$ & $42: 32: 17.14$ & 6.4 & NVSS J202540+423222 & Condon et al. (1998) & \\
\hline- & 1569 & - & $20: 25: 42.91$ & $41: 56: 15.55$ & 5.6 & NVSS J202543+415618 & Vollmer et al. (2010) & \\
\hline- & 1572 & - & $20: 25: 44.10$ & $41: 56: 02.14$ & 6.0 & $\mathrm{~J} 202544.53+415605.70$ & Kryukova et al. (2014) & YSO \\
\hline 0600 & 1680 & $-1.0 \pm 0.1$ & $20: 26: 25.48$ & $42: 32: 09.42$ & 5.2 & NVSS J202625+423214 & Condon et al. (1998) & \\
\hline 0604 & 1685 & $-0.8 \pm 0.1$ & $20: 26: 26.26$ & $44: 39: 27.16$ & 3.9 & NVSS J202625+443927 & Vollmer et al. (2010) & \\
\hline 0626 & 1728 & $-0.9 \pm 0.1$ & $20: 26: 42.98$ & $40: 51: 28.03$ & 10.5 & NVSS J202642+405138 & Vollmer et al. (2010) & \\
\hline- & 1821 & - & $20: 27: 18.99$ & $40: 25: 00.97$ & 1.0 & WSRTGP 2025+4015 & Vollmer et al. (2010) & \\
\hline 0684 & 1822 & $-1.1 \pm 0.1$ & $20: 27: 19.57$ & $43: 13: 58.44$ & 14.3 & TYC 3164-341-1 & Høg et al. (2000) & Star \\
\hline 0722 & 1908 & $-0.8 \pm 0.1$ & $20: 27: 52.92$ & $41: 35: 05.03$ & 5.2 & $\mathrm{~J} 202753.37+413506.03$ & Kryukova et al. (2014) & IR source \\
\hline 0735 & 1944 & $-0.4 \pm 0.1$ & $20: 28: 04.06$ & $41: 13: 54.24$ & 5.1 & $\mathrm{~J} 202804.51+411354.56$ & Kryukova et al. (2014) & YSO \\
\hline 0744 & 1957 & $-0.8 \pm 0.1$ & 20:28:07.20 & $41: 13: 50.64$ & 7.9 & UVEX J202807.55+411357.7 & Verbeek et al. (2012) & White dwarf \\
\hline- & 1967 & - & $28: 28: 10.38$ & $45: 12: 51.73$ & 0.2 & NVSS J202810+451251 & Condon et al. (1998) & \\
\hline- & 1980 & - & $20: 28: 13.29$ & $40: 16: 52.83$ & 3.6 & WSRTGP 2026+4006 & Vollmer et al. (2010) & \\
\hline 0769 & 2013 & $-1.1 \pm 0.1$ & $20: 28: 24.34$ & $40: 37: 50.03$ & 3.9 & $19 \mathrm{P} 6$ & Wendker et al. (1991) & \\
\hline- & 2027 & - & $20: 28: 31.01$ & 40:59:59.05 & 3.9 & J202831.35+405959.24 & Kryukova et al. (2014) & YSO \\
\hline 0788 & 2068 & $-1.1 \pm 0.1$ & $20: 28: 49.60$ & $41: 18: 37.36$ & 0.6 & TYC 3160-1079-1 & Høg et al. (2000) & Star \\
\hline 0789 & 2070 & $-1.3 \pm 0.1$ & $20: 28: 50.79$ & $41: 34: 37.13$ & 5.5 & NVSS J202851+413438 & Vollmer et al. (2010) & \\
\hline 0804 & 2108 & $-1.1 \pm 0.1$ & 20:29:00.90 & $42: 12: 59.28$ & 5.5 & NVSS J202901+421259 & Condon et al. (1998) & \\
\hline 0806 & 2115 & $-0.9 \pm 0.1$ & 20:29:04.20 & 41:00:05.78 & 4.1 & $19 \mathrm{P} 7$ & Wendker et al. (1991) & \\
\hline 0807 & 2118 & $-1.0 \pm 0.1$ & 20:29:04.70 & $42: 35: 27.90$ & 4.4 & TYC 3160-1447-1 & Høg et al. (2000) & Star \\
\hline- & 2159 & - & $20: 29: 23.53$ & 40:11:09.57 & 4 & AFGL 2591 & Johnston et al. (2013) & Star-forming region \\
\hline- & 2170 & - & $20: 29: 28.60$ & $40: 57: 24.41$ & 7.0 & $19 \mathrm{P} 9$ & Wendker et al. (1991) & \\
\hline- & 2174 & - & $20: 29: 30.94$ & $41: 34: 20.72$ & 5.2 & J202931.39+413421.96 & Kryukova et al. (2014) & YSO \\
\hline- & 2189 & - & $20: 29: 36.50$ & $41: 51: 40.89$ & 4.6 & WSRTGP 2027+4141 & Vollmer et al. (2010) & \\
\hline
\end{tabular}


Table A.1. continued.

\begin{tabular}{|c|c|c|c|c|c|c|c|c|}
\hline $\begin{array}{l}325-I D \\
\text { BIC325- }\end{array}$ & $\begin{array}{r}\text { 610-ID } \\
\text { BIC610- }\end{array}$ & $\begin{array}{l}\text { Spectral } \\
\text { index }\end{array}$ & $\begin{array}{l}\mathrm{RA}_{\mathrm{J} 2000} \\
(\mathrm{~h}, \mathrm{~m}, \mathrm{~s})\end{array}$ & $\begin{array}{l}\operatorname{Dec}_{J 2000} \\
\left({\text { deg, },,^{\prime \prime}}^{\prime}\right) \\
\end{array}$ & $\begin{array}{c}d \\
\left({ }^{\prime \prime}\right)\end{array}$ & $\begin{array}{l}\text { SIMBAD nearest } \\
\text { source }\end{array}$ & Reference & $\begin{array}{l}\text { Information on } \\
\text { possible nature }\end{array}$ \\
\hline 0846 & 2215 & $-1.7 \pm 0.3$ & $20: 29: 49.42$ & 41:43:13.66 & 16.7 & ** GRV 344 & Greaves (2004) & Stellar system \\
\hline- & 2223 & - & $20: 29: 52.06$ & $40: 48: 45.82$ & 2.7 & G079.4430+01.0047 & Urquhart et al. (2009) & HII region \\
\hline 0854 & - & - & $20: 29: 59.18$ & $41: 16: 44.20$ & 7.0 & IRAS 20286+4105 & Ramachandran et al. (2017) & Star-forming region \\
\hline 0867 & 2280 & $-0.9 \pm 0.1$ & $20: 30: 14.17$ & $40: 41: 08.67$ & 10.4 & IRAS 20283+4031 & Parthasarathy et al. (1992) & Star \\
\hline 0872 & 2299 & $-1.2 \pm 0.1$ & $20: 30: 30.47$ & $44: 12: 26.40$ & 4.9 & 2MASS J20303018+4412301 & Price-Whelan et al. (2018) & Star \\
\hline 0873 & 2311 & $-0.7 \pm 0.1$ & $20: 30: 36.58$ & $41: 06: 06.10$ & 6.2 & NVSS J203032+410634 & Williams et al. (2013) & \\
\hline - & 2313 & - & $20: 30: 37.68$ & $42: 20: 57.96$ & 15.9 & WSRTGP 2028+4211 & Vollmer et al. (2010) & \\
\hline 0874 & 2319 & $-0.8 \pm 0.1$ & $20: 30: 39.72$ & $41: 23: 31.73$ & 0.4 & NVSS J203039+412331 & Vollmer et al. (2010) & \\
\hline- & 2351 & - & $20: 30: 57.60$ & $43: 08: 05.33$ & 6.9 & WSRTGP 2029+4257 & Vollmer et al. (2010) & \\
\hline 0882 & 2370 & $-2.1 \pm 0.3$ & $20: 31: 10.22$ & $40: 58: 53.80$ & 2.5 & $\mathrm{~J} 203110.44+405853.94$ & Kryukova et al. (2014) & YSO \\
\hline- & 2376 & - & $20: 31: 14.29$ & $42: 22: 42.95$ & 4.1 & TYC 3161-82-1 & Høg et al. (2000) & Star \\
\hline 0889 & 2381 & $-1.0 \pm 0.2$ & $20: 31: 18.69$ & $41: 09: 25.16$ & 10.7 & RLP 933 & Reddish et al. (1966) & Star \\
\hline - & 2382 & - & $20: 31: 19.14$ & $40: 18: 09.90$ & 0.4 & IRAS $20293+4007$ VLA 3 & Sánchez-Monge et al. (2008) & \\
\hline 0890 & 2384 & $-1.0 \pm 0.1$ & $20: 31: 19.90$ & $40: 40: 56.04$ & 1.6 & GPSR $079.501+0.704$ & Zoonematkermani et al. (1990) & \\
\hline- & 2406 & - & $20: 31: 37.33$ & $40: 22: 58.79$ & 0.3 & G79.29+0.46 & Higgs et al. (1994) & Wind shell \\
\hline- & 2409 & - & $20: 31: 39.72$ & 40:16:08.36 & 0.2 & G79.29+0.46 & Higgs et al. (1994) & Wind shell \\
\hline- & 2420 & - & $20: 31: 51.59$ & $41: 31: 18.60$ & 2.8 & BDB2006-234 & Paredes et al. (2008) & X-ray star \\
\hline 0906 & 2432 & $-1.4 \pm 0.1$ & $20: 32: 00.50$ & $41: 36: 58.45$ & 1.0 & J203201.7+413722 & Paredes et al. (2008) & Galaxy S lobe \\
\hline 0908 & 2435 & $-1.4 \pm 0.1$ & 20:32:01.91 & $41: 37: 47.43$ & 1.0 & $\mathrm{~J} 203201.7+413722$ & Paredes et al. (2008) & Galaxy N lobe \\
\hline 0915 & 2448 & $-2.1 \pm 0.4$ & $20: 32: 12.92$ & $41: 27: 24.01$ & 2.3 & MT91-213 & Chen et al. (2019) & Be star+pulsar \\
\hline - & 2452 & - & $20: 32: 14.13$ & $40: 42: 24.88$ & 0.7 & NVSS J203214+404226 & Vollmer et al. (2010) & \\
\hline - & 2463 & - & $20: 32: 21.17$ & $40: 17: 18.63$ & 0.5 & DR $15 \mathrm{I}$ & Colley (1980) & Part of nebula \\
\hline 0921 & 2464 & $-0.5 \pm 0.2$ & $20: 32: 22.32$ & $41: 18: 19.28$ & 1.2 & Cyg OB2 5 & De Becker \& Raucq (2013) & OB stellar system \\
\hline 0924 & 2469 & $+1.4 \pm 0.1$ & $20: 32: 25.65$ & $40: 57: 28.09$ & 1.5 & WR 145a & Bulgarelli et al. (2019) & HMXB \\
\hline 0925 & 2470 & $-0.5 \pm 0.1$ & $20: 32: 26.77$ & $41: 04: 33.28$ & 1.3 & HSC N & Martí et al. (2006) & Part of cloud \\
\hline 0927 & 2472 & $-1.3 \pm 0.2$ & $20: 32: 29.25$ & 41:35:07.36 & 1.7 & 2MASS J20322935+4135061 & Cutri et al. (2003) & IR source \\
\hline 0928 & 2473 & $-0.1 \pm 0.3$ & $20: 32: 29.52$ & $40: 38: 49.65$ & 0.9 & GPSR $079.602+0.506$ & Zoonematkermani et al. (1990) & \\
\hline 0933 & - & - & $20: 32: 36.60$ & $41: 14: 47.87$ & 14.4 & RLP 886 & Reddish et al. (1966) & Star \\
\hline - & 2494 & - & $20: 32: 40.83$ & $41: 14: 29.31$ & 1.4 & Cyg OB2 12 & De Becker \& Raucq (2013) & Star \\
\hline 0941 & 2501 & $+0.6 \pm 0.1$ & $20: 32: 45.44$ & $40: 39: 37.50$ & 1.3 & 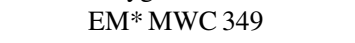 & Zhang et al. (2017) & Emission-line star \\
\hline 0946 & 2514 & $-0.9 \pm 0.1$ & $20: 32: 55.32$ & 40:31:30.99 & 4.6 & $19 \mathrm{P} 22$ & Wendker et al. (1991) & \\
\hline 0953 & 2526 & $-1.1 \pm 0.1$ & $20: 33: 10.27$ & $40: 41: 16.91$ & 1.9 & $\mathrm{~J} 203310.31+404118.72$ & Kryukova et al. (2014) & YSO \\
\hline " & 2528 & " & $20: 33: 11.01$ & $40: 41: 32.23$ & 4.8 & $19 \mathrm{P} 24$ & Vollmer et al. (2010) & \\
\hline " & 2530 & " & 20:33:11.74 & $40: 41: 49.35$ & 2.1 & $\mathrm{~J} 203311.80+404151.32$ & Kryukova et al. (2014) & YSO \\
\hline- & 2536 & - & $20: 33: 14.93$ & $41: 18: 50.63$ & 1.7 & Cyg OB2 8A & De Becker \& Raucq (2013) & OB stellar system \\
\hline 0956 & $2544 / 2545$ & $-0.3 \pm 0.1$ & 20:33:18.92 & $40: 58: 37.39$ & 14.4 & G079.964+00.579 & Anderson et al. (2015) & \\
\hline 0957 & 2545 & $-0.4 \pm 0.1$ & 20:33:19.00 & 40:59 05.06 & 13.6 & G079.964+00.579 & Anderson et al. (2015) & \\
\hline " & - & - & 20:33:19.00 & $40: 5905.06$ & 15.5 & BD+40 4230 & Reddish et al. (1966) & Star \\
\hline 0963 & $2552 / 2556 / 2558$ & $-1.2 \pm 0.1$ & $20: 33: 23.42$ & $41: 27: 17.60$ & 6.6 & 19P 26 & Vollmer et al. (2010) & \\
\hline & & & $20: 33: 23.42$ & $41: 27: 17.60$ & 22.3 & IDX 114 & Rauw (2011) & X-ray star \\
\hline 0965 & 2559 & $-0.8 \pm 0.3$ & $20: 33: 24.95$ & $40: 57: 28.81$ & 8.3 & RLP 1034 & Reddish et al. (1966) & Star \\
\hline 0966 & 2560 & $-0.5 \pm 0.3$ & $20: 33: 26.58$ & $40: 42: 33.04$ & 1.8 & $\mathrm{~J} 203326.44+404233.74$ & Kryukova et al. (2014) & YSO \\
\hline - & 2565 & - & $20: 33: 30.74$ & $41: 35: 28.42$ & 8.5 & RLP 283 & Reddish et al. (1966) & Star \\
\hline 0968 & 2566 & $-0.3 \pm 0.1$ & $20: 33: 31.97$ & 40:41:03.03 & 8.2 & $19 \mathrm{P} 28$ & Wendker et al. (1991) & \\
\hline 0976 & 2585 & $-1.4 \pm 0.1$ & $20: 33: 47.12$ & $40: 40: 54.64$ & 10.6 & $\mathrm{~J} 203348.01+404051.61$ & Kryukova et al. (2014) & YSO \\
\hline 0977 & 2589 & $-0.9 \pm 0.1$ & $20: 33: 52.22$ & $41: 15: 45.11$ & 7.7 & AFM2007-990 & Albacete Colombo et al. (2007) & $\mathrm{X}$-ray star \\
\hline- & 2603 & - & 20:34:06.69 & 41:16:00.86 & 1.0 & J203406.77+411600.46 & Kryukova et al. (2014) & YSO \\
\hline 0985 & 2607 & $-0.3 \pm 0.2$ & $20: 34: 10.56$ & 41:06:58.71 & 0.7 & IPHASX J203410.5+410659 & Wright et al. (2012) & frEGG \\
\hline 0988 & 2610 & $-2.5 \pm 0.2$ & $20: 34: 13.85$ & 41:08:16.31 & 5.0 & WDDGGHK7 & Isequilla et al. (2019) & frEGG \\
\hline 1000 & 2635 & $-1.6 \pm 0.2$ & $20: 34: 36.48$ & $40: 51: 59.24$ & 5.3 & WDDGGHK4 & Isequilla et al. (2019) & frEGG \\
\hline 1003 & 2638 & $-1.3 \pm 0.1$ & $20: 34: 43.28$ & $40: 53: 15.48$ & 2.1 & WDDGGHK3 & Isequilla et al. (2019) & frEGG \\
\hline- & 2652 & - & $20: 34: 53.33$ & $40: 53: 20.89$ & 2.2 & WDDGGHK2 & Isequilla et al. (2019) & frEGG \\
\hline 1006 & 2641 & $-0.8 \pm 0.3$ & $20: 34: 45.15$ & $41: 45: 03.2$ & 3.2 & GPSR $079.918+0.283$ & Zoonematkermani et al. (1990) & \\
\hline - & 2625 & — & $20: 34: 29.54$ & $41: 31: 45.2$ & 0.7 & TYC 3161-1048-1 & Høg et al. (2000) & star \\
\hline 1012 & 2654 & $-0.6 \pm 0.1$ & $20: 34: 55.80$ & $40: 40: 46.56$ & 1.6 & GPSR $079.904+0.154$ & Zoonematkermani et al. (1990) & \\
\hline - & 2657 & - & $20: 34: 57.30$ & 40:04:14.94 & 6.5 & TYC 3157-1182-1 & Høg et al. (2000) & Star \\
\hline 1014 & 2659 & $+0.4 \pm 0.1$ & $20: 35: 00.28$ & $41: 34: 52.90$ & 2.34 & IRAS $20332+4124$ & Lu et al. (2014) & Star-forming region \\
\hline 1018 & 2663 & $-0.0 \pm 0.2$ & $20: 35: 02.77$ & $41: 34: 51.22$ & 10.8 & IRAS $20332+4124$ & Lu et al. (2014) & Star-forming region \\
\hline 1019 & 2664 & $-0.1 \pm 0.2$ & 20:35:03.56 & $41: 18: 24.12$ & 0.4 & 2MASS J20350353+4118240 & Cutri et al. (2003) & IR source \\
\hline - & 2666 & - & 20:35:07.84 & $39: 59: 48.46$ & 2.8 & GPSR $079.380-0.284$ & Zoonematkermani et al. (1990) & \\
\hline 1020 & 2670 & $+0.9 \pm 0.1$ & $20: 35: 16.63$ & $40: 49: 44.66$ & 1.0 & GPSR 080.063+0.191 & Zoonematkermani et al. (1990) & \\
\hline 1024 & 2680 & $-1.2 \pm 0.1$ & $20: 35: 32.37$ & $41: 44: 56.33$ & 0.8 & NVSS J203532+414456 & Condon et al. (1998) & \\
\hline
\end{tabular}


P. Benaglia et al.: Cygnus survey with the Giant Metrewave Radio Telescope at 325 and $610 \mathrm{MHz}$ : the catalog

Table A.1. continued.

\begin{tabular}{|c|c|c|c|c|c|c|c|c|}
\hline $\begin{array}{l}\text { 325-ID } \\
\text { BIC325- }\end{array}$ & $\begin{array}{c}\text { 610-ID } \\
\text { BIC610- }\end{array}$ & $\begin{array}{l}\text { Spectral } \\
\text { index }\end{array}$ & $\begin{array}{l}\mathrm{RA}_{\mathrm{J} 2000} \\
(\mathrm{~h}, \mathrm{~m}, \mathrm{~s})\end{array}$ & $\begin{array}{l}\operatorname{Dec}_{J 2000} \\
\left(\operatorname{deg}^{\prime},{ }^{\prime},{ }^{\prime}\right)\end{array}$ & $\begin{array}{c}d \\
\left({ }^{\prime \prime}\right)\end{array}$ & $\begin{array}{l}\text { SIMBAD nearest } \\
\text { source }\end{array}$ & Reference & $\begin{array}{l}\text { Information on } \\
\text { possible nature }\end{array}$ \\
\hline 1025 & - & - & $20: 35: 33.17$ & $41: 06: 45.07$ & 2.8 & GPSR $080.322+0.319$ & Vollmer et al. (2010) & \\
\hline 1027 & - & - & $20: 35: 42.72$ & $40: 52: 51.58$ & 1.7 & GPSR $080.154+0.156$ & Zoonematkermani et al. (1990) & \\
\hline 1030 & 2689 & $+0.2 \pm 0.2$ & 20:35:47.07 & $41: 22: 45.01$ & 0.4 & WR 146 & De Becker \& Raucq (2013) & WR system \\
\hline — & 2693 & - & $20: 35: 55.32$ & 42:18:03.67 & 3.5 & $2034+42 \mathrm{~A}$ & Clegg et al. (1992) & \\
\hline - & 2697 & - & $20: 35: 58.48$ & $42: 17: 23.86$ & 3.1 & $2034+42 B$ & Clegg et al. (1992) & \\
\hline 1032 & 2698 & $-0.6 \pm 0.2$ & $20: 35: 59.52$ & 40:54:00.86 & 12.5 & $\mathrm{~J} 203558.60+405353.85$ & Kryukova et al. (2014) & YSO \\
\hline$"$ & " & " & 20:36:01.31 & $40: 53: 56.79$ & 4.4 & $\mathrm{~J} 203600.94+405358.24$ & Kryukova et al. (2014) & YSO \\
\hline " & 2700 & " & $20: 36: 01.31$ & $40: 53: 56.79$ & 8.1 & J203601.85+405351.48 & Kryukova et al. (2014) & YSO \\
\hline 1034 & 2707 & $+0.2 \pm 0.2$ & $20: 36: 12.84$ & $40: 45: 43.14$ & 2.1 & GPSR $080.115+0.009$ & Zoonematkermani et al. (1990) & \\
\hline 1038 & 2721 & $-0.6 \pm 0.1$ & $20: 36: 29.72$ & $41: 20: 21.90$ & 0.5 & CPR2002-B3 & Comerón et al. (2002) & Star \\
\hline 1039 & 2722 & $-1.2 \pm 0.1$ & $20: 36: 34.45$ & $41: 32: 22.66$ & 10.9 & G080.522+00.714 & Zoonematkermani et al. (1990) & \\
\hline - & 2730 & - & $20: 36: 43.72$ & 40:21:09.99 & 2.6 & WR 147 & De Becker \& Raucq (2013) & WR system \\
\hline - & 2770 & - & $20: 37: 37.72$ & $40: 53: 52.54$ & 1.8 & $080.386-0.122$ & Garwood et al. (1988) & \\
\hline - & 2785 & - & $20: 37: 58.29$ & $40: 00: 52.85$ & 2.9 & $18 \mathrm{P} 6$ & Vollmer et al. (2010) & \\
\hline - & 2793 & - & $20: 38: 22.23$ & $40: 16: 16.95$ & 2.0 & GPSR 079.972-0.614 & Zoonematkermani et al. (1990) & \\
\hline
\end{tabular}

